\title{
ÍNDICES DE CAMBIO CLIMÁTICO EN LA RESERVA DE LA BIOSFERA EL VIZCAÍNO, BAJA CALIFORNIA SUR, MÉXICO (1960-2012)
}

\author{
KACHOK, René - rkachok@cibnor.mx \\ Centro de Investigaciones Biológicas del Noroeste / México
}

IVANOVA, Antonina - aivanova@uabcs.mx

Universidade Autonoma de Baja California Sur / México

\begin{abstract}
RESUMO: Este artigo determina a tendência dos índices climáticos com base nas temperaturas máximas e mínimas e precipitação registrada em estações meteorológicas no El Vizcaíno Reserva da Biosfera (REBIVI), Baja California Sur, no México, no período de 1960 a 2012. Para tanto, foi usada uma série de dados históricos de 9 estações meteorológicas distribuídas em REBIVI. Foram analisados 27 índices de mudanças climáticas determinados pelo Painel sobre detecção e Indicadores de Mudanças Climáticas (Equipe de Peritos sobre Detecção de Mudanças Climáticas e índices, ETCCDI), onde 16 deles estão relacionados com temperatura e precipitação. De forma a complementar e confirmar as os pontos de mudanças encontrados, foi primeiramente aplicado um teste de homogeneidade RHtestsV4 para a temperatura máxima e mínima, e posteriormente aplicado o teste RHtests_dlyPrcp para a precipitação. O programa ClimDex desenvolvido pela National Oceanic and Atmospheric Administration (NOAA) foi usado com uma plataforma de programa estatístico de R. Dos 27 índices propostos pela ETCCDI decidiu-se analisar apenas 16 . No período de 50 anos, $75 \%$ desses índices apresentaram uma tendência de aumento, e 25\% mostraram uma tendência de declínio.
\end{abstract}

PALAVRAS-CHAVE: índices climáticos, temperaturas máximas e mínimas, precipitação, El Vizcaíno Reserva da Biosfera, México.

\section{CLIMATE CHANGE INDEXES AT THE BIOSPHER RESERV OF EL VIZCAÍNO, BAJA CALIFORNIA SUR, MÉXICO (1960-2012)}

\begin{abstract}
This article determines the trend of climatic indexes based on maximum and minimum temperatures and precipitation recorded at the meteorological stations in the El Vizcaino Biosphere Reserve (REBIVI), state of Baja California Sur, Mexico, during the period from 1960 to 2012. The historical data series of 9 climatological stations distributed in the REBIVI were used. We evaluated 27 climate change indices determined by the Expert Group on Climate Change Detection and Indicators (ETCCDI), where 16 of them are related to temperature and eleven with precipitation. In order to complement and corroborate the found points of change, a firstly, a homogeneity test RHtestsV4 was applied for maximum and minimum temperature, and secondly RHtests_dlyPrcp was applied for precipitation. We used the ClimDex program, developed by the National Oceanic and Atmospheric Administration (NOAA), with a platform the statistical program of R. Of the 27 indexes proposed by the ETCCDI, it was decided to use for this analysis only 16 . In the period of fifty years, $75 \%$ of the indices showed a tendency to increase, and $25 \%$ showed a tendency to decrease.
\end{abstract}

KEYWORDS: climatic indexes, maximum and minimum temperatures, precipitation, El Vizcaino Biosphere Reserve, Mexico

\section{INTRODUCCIÓN}

En el año 2003, la Organización Meteorológica Mundial (OMM) anunció la posibilidad de un incremento en los extremos del tiempo y el clima asociado al calentamiento global. Más tarde el cuarto y quinto informes de evaluación del grupo Intergubernamental de Expertos sobre el Cambio Climático (IPCC 2007, 2012) reporta evidencias sustanciales de que el calentamiento del sistema climático es inequívoco. Tales afirmaciones son sustentadas por investigaciones recientes sobre detección de cambios en extremos climáticos a escala global y 
regional (Alexander et al. 2006). En las mismas se han encontrado evidencias de cambios en correspondencia con el aumento de la temperatura del planeta, incremento en los eventos de temperaturas altas, disminución de eventos de temperaturas bajas e incremento en la lluvia intensa.

En un estudio realizado por Rodríguez-Moreno et al. (1978), menciona que el cambio climático es ya uno de los problemas a nivel global. De acuerdo al Panel Intergubernamental de Cambio Climático (IPCC 2007), durante los últimos 100 años la temperatura en el planeta se ha aumentado en más o menos $0.74^{\circ} \mathrm{C}$. Yu et al. (2015) mencionan que en los últimos 30 años los periodos de calor estuvieron por encima del promedio, por lo que se espera que la frecuencia y la intensidad de las temperaturas extremas se incrementen. El impacto de los climas extremos se manifiesta a través de las inundaciones, el fuego, las sequías y las ondas de calor.

Este impacto ocurre por lo general cuando los climas extremos se presentan con cierta regularidad e intensidad, provocando desastres ecológicos y afectando económicamente a la sociedad por las pérdidas en sus bienes 0 pertenencias (Degefie et al. 2014). Muchos estudios atribuyen que una de las principales causas del incremento en la temperatura promedio a nivel global es debida directamente a la generación de gases efecto invernadero (GEI) ocasionadas de manera antropogénica, es decir, por las actividades realizadas por los seres humanos (industria, generación de energía, vehículos, deforestación, cambio de usos de suelo, ganadería, entre otras) (IPCC 2007). El calentamiento de la superficie de los océanos, el aumento de la temperatura continental, la temperatura extrema y los patrones de comportamiento de los vientos son influenciados por este fenómeno y puede resultar en extremos sin precedente del clima (IPCC 2012).

Por otro lado, los procesos biogeoquímicos como el caso del ciclo del carbón y el nitrógeno pueden ser controlados por las ondas de calor, lo cual puede modificar la función de estos procesos afectando el ecosistema (Degefie et al. 2014). Arnone et al. (2008) observaron que el aumento en la frecuencia de años cálidos, disminuye la captación del dióxido de carbono (CO2) del ecosistema terrestre. Otros estudios recientes mencionan una tendencia de disminución en cuanto a la frecuencia de extremos fríos y el aumento de la temperatura tanto diurna como nocturna (Acquaotta et al. 2010, Wang et al. 2013). Existen estudios que muestran que en los últimos 50 años ha habido una disminución en el número de noches frías y el número de noches cálidas va en aumento en $70 \%$ del planeta (Alexander et al. 2006).

El IPCC (2007) mencionó la vulnerabilidad de México ante el cambio climático, remarcando que en la región del noroeste de México y el suroeste de Estados Unidos de América, el problema de la escasez de agua puede agravarse debido al aumento en la temperatura y a la escasez de lluvias (Cavazos y Arriaga-Ramírez 2012). En un estudio de Magaña et al. (2012) las regiones que pueden presentar riesgo por el aumento de la temperatura promedio anual se espera que sean en la región noreste y noroeste de México (Magaña et al. 2012).

Sin embargo, diversos estudios sobre las temperaturas extremas en México, mencionan que a partir del año de 1970, se presentó un aumento significativo en la temperatura máxima en comparación con las temperaturas mínimas, especialmente en las regiones noroeste y central de México, lo cual 
mostró tendencias contrastantes con respecto al resto del país (Englehart y Douglas 2005, Pavía et al. 2008, Gutierrez-Ruacho et al., 2010).

García y Mosiño (1968), mencionan que la ubicación del noroeste de México en el extremo occidental de la gran masa continental de Norteamérica tiene dos consecuencias notables que condicionan en gran medida su aridez. En primer lugar, está sometida a los efectos de la celda de alta presión del Pacífico durante la mayor parte del año; y en segundo término, la influencia de la corriente de California que discurre de Norte a Sur paralela a la costa de la península de Baja California (tomado de INECC, http://www2.inecc.gob.mx/publicaciones/libros/451/cap3.html).

La corriente oceánica fría de California, modifica y controla el régimen meteorológico general, sobre todo a bajas altitudes, aumentando la zona atmosférica que inhibe la formación de nubes de tipo convectivo sobre las cercanías de la costa del Pacífico (Álvarez 1995).

De acuerdo al sistema de clasificación de Köppen (1948, en Rzedowski 1978) a la península de Baja California le corresponde la clasificación de clima tipo B, o seco, que cubre la mayor parte de México, sobre todo en la mitad septentrional de su territorio y en particular, en el altiplano, planicies costeras noroccidentales y nororientales. Los climas de categoría BW (seco desértico), predominan en la mayor parte de la península y en menor medida encontramos la categoría Bs (seco estepario). Los climas de tipo $\mathrm{C}$, o templados y húmedos, son característicos de las zonas de montaña de México. La categoría Cs (con temporada lluviosa en época fría del año), es propia de las montañas de Baja California.

El propósito del presente estudio es con la finalidad de determinar las tendencias de los índices climáticos, basados en las temperaturas máximas, temperaturas mínimas y precipitación registradas en las estaciones meteorológicas de la Reserva de la Biosfera El Vizcaíno, Mulegé, B.C.S., en el periodo de 1960 a 2012.

\section{MATERIALES Y MÉTODOS}

Datos generales de la Reserva de la Biosfera El Vizcaíno, en el Municipio de Mulegé, Baja California Sur.

- Se decretó Reserva de la Biosfera el 30 de noviembre de 1988 (CONANP 2000).

-En 1993 el Comité del Patrimonio Mundial de la Unesco, inscribió a las "Pinturas Rupestres de la Sierra de San Francisco" y como "Refugio de Ballenas en las Lagunas del Vizcaíno" a las lagunas Ojo de Liebre y San Ignacio, por su valor excepcional y universal como sitios cultural y natural, respectivamente, que deben ser protegidos por la humanidad (CONANP 2000).

- La superficie de la Reserva de la Biosfera El Vizcaíno es de 2546790 ha, representando el $76.96 \%$ de la superficie del municipio de Mulegé (3 309 220 ha), Baja California Sur (CONANP 2000). 
- La zona de amortiguamiento tiene una superficie de 183351 ha y la zona núcleo 363439 ha (CONANP 2000).

- La Reserva incluye $5 \mathrm{~km}$ de franja costera de Mar Territorial de ambas costas con el fin de incluir la ruta migratoria de la ballena gris, las islas y los diferentes recursos pesqueros existentes (CONANP 2000).

- La superficie del Patrimonio de la Humanidad "Pinturas Rupestres de la Sierra de San Francisco" es de 182600 ha (UNESCO 2016).

- La superficie del Patrimonio de la Humanidad "Refugio de Ballenas en las Lagunas del Vizcaíno" es de 369631 ha (UNESCO 2016).

En la Figura 1 se muestra el área de la REBIVI (color verde) y las áreas núcleo (color rojo).

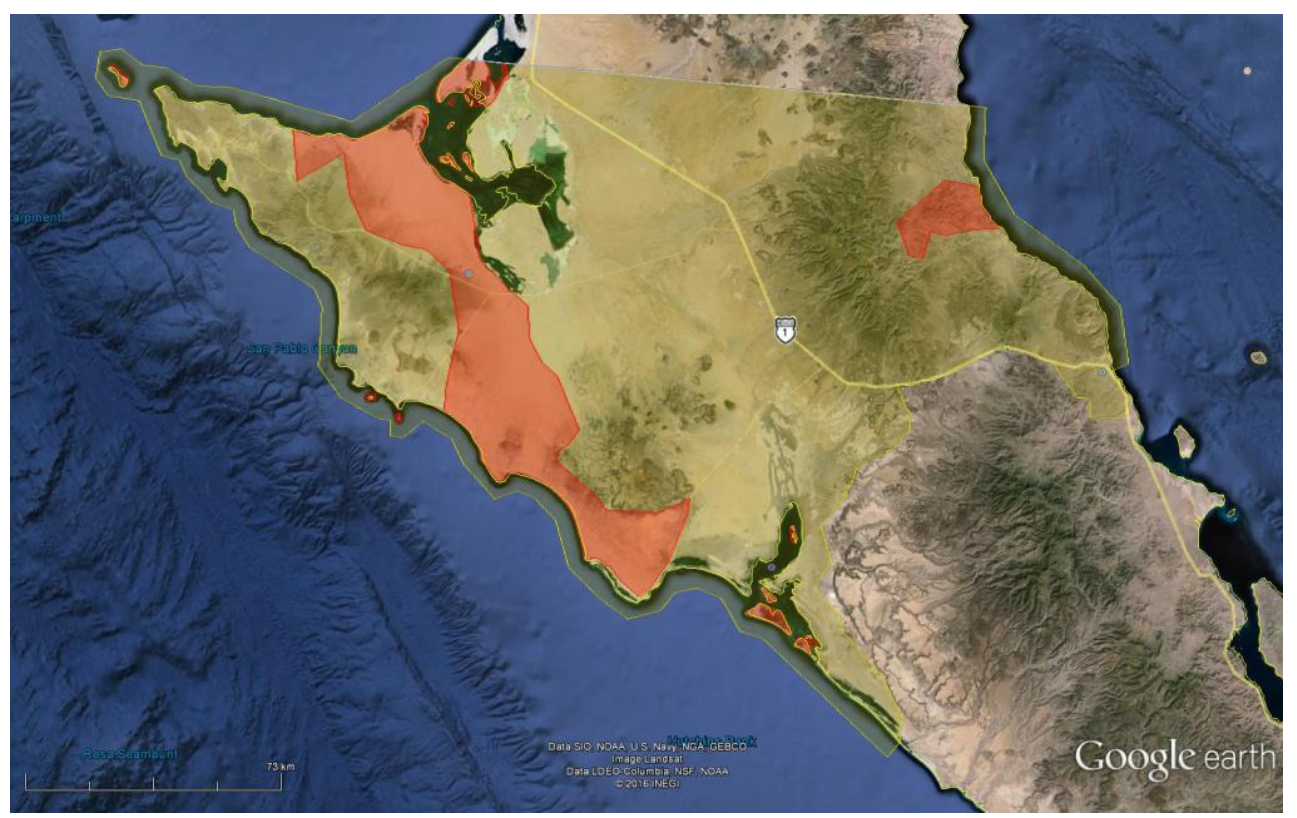

FIGURA 1 - Área de la Reserva de la Biosfera El Vizcaíno y sus zonas núcleo. FUENTE: Google earth, y datos de INEGI, México.

Para la realización del presente estudio se utilizó la información de 9 estaciones meteorológicas de la Comisión Nacional del Agua (CONAGUA) distribuidas en la REBIVI, con registros de datos diarios de temperatura máxima, temperatura mínima y precipitación de los años de 1960 a 2012 . En la Figura 2 se muestra la localización de las estaciones meteorológicas. 


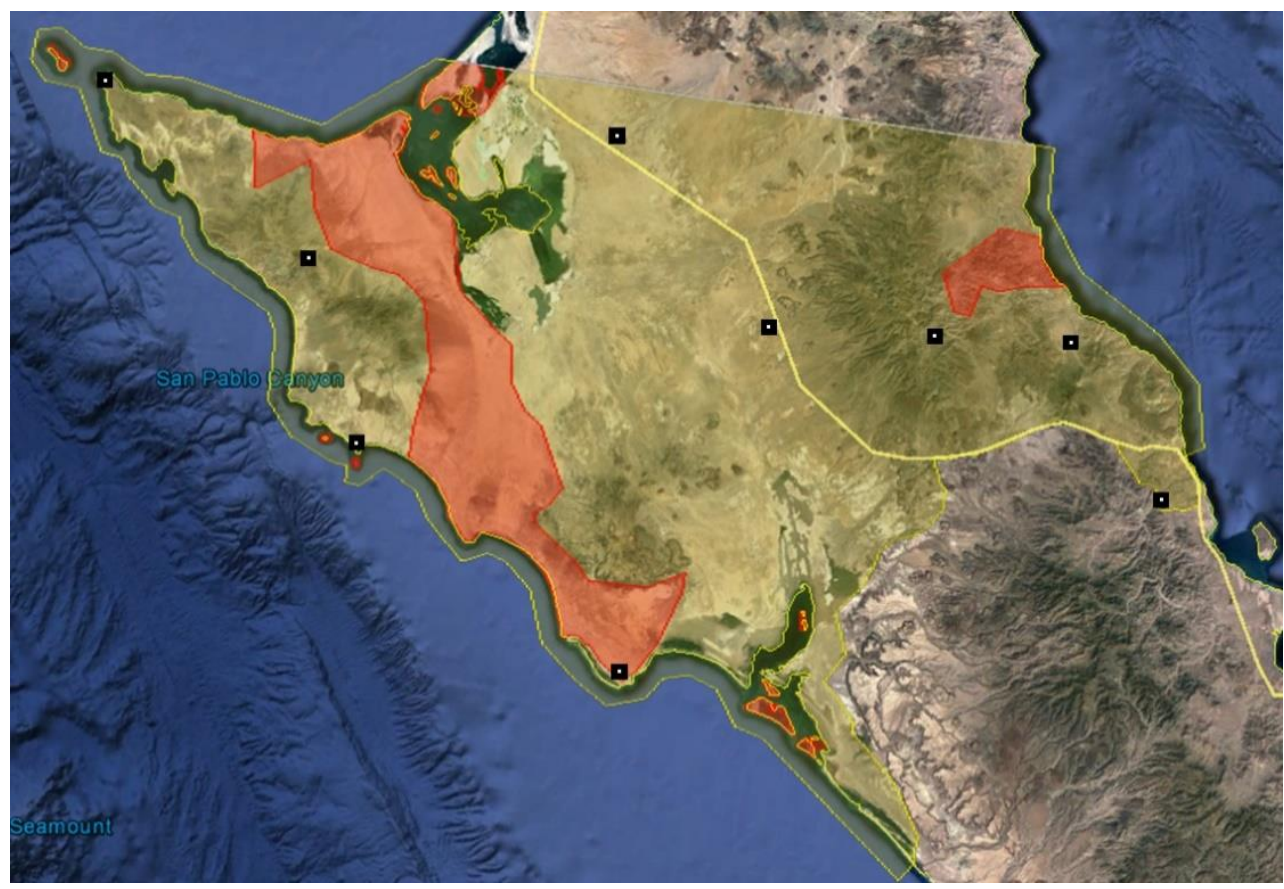

FIGURA 2 - Estaciones meteorológicas en la REBIVI, B.C.S.Fuente: Google Earth, y CNA, México.

\section{CALIDAD Y HOMOGENEIDAD DE LAS SERIES}

A los datos obtenidos de las 9 estaciones meteorológicas se les aplicó un control de calidad, para ello se utilizó el programa RClimDex v. 01-5, ejecutado en el programa estadístico R v. 3.0.1., (Zhang y Feng 2004). También se realizó un análisis de homogeneidad y la detección de saltos o cambios abruptos en las series de los índices analizados de acuerdo a Wang (2008), se utilizó la prueba de $F$ de máxima penalización utilizando el módulo RHtestsV4 para la temperatura máxima y temperatura mínima, y para la precipitación se aplicó RHtests_dlyPrcp, para cambios en la media en datos no documentados (Wang y Feng, 2010). En el RHTest se realizó también el proceso de homogeneización de la serie de datos con ajuste por cuantiles (QM), en los casos donde los datos no resultaron homogéneos (Wang y Feng, 2010). Una vez aplicado los análisis de calidad a la serie de datos, a los índices obtenidos se les realizó un análisis de estadística descriptiva para determinar anomalías en las variables térmicas o de temperatura y de precipitación.

Para la determinación de los índices climáticos, se seleccionaron 16 de los 27 índices de cambio climático establecidos por el equipo de expertos para la detección, monitoreo e índices de cambio climático" (ETCCDI) (Vázquez 2010, Zhang et al. 2015). Los índices climáticos seleccionados fueron los relacionados con la temperatura y la precipitación (Tabla 1).

TABLA 1 - Índices de cambio climático para la Reserva de la Biosfera 


\begin{tabular}{|c|c|c|c|}
\hline ID & $\begin{array}{l}\text { Nombre del } \\
\text { Indicador }\end{array}$ & Definición & $\begin{array}{l}\text { UNIDA } \\
\text { D }\end{array}$ \\
\hline CWD & $\begin{array}{l}\text { Consecutive wet days } \\
\text { (Días húmedos } \\
\text { consecutivos) }\end{array}$ & $\begin{array}{l}\text { Número máximo de días } \\
\text { consecutivos } \\
\text { RR }>=1 \mathrm{~mm}\end{array}$ & Días \\
\hline $\mathrm{R} 20 \mathrm{~mm}$ & $\begin{array}{l}\text { Number of very heavy } \\
\text { precipitation days } \\
\text { (Número de días con } \\
\text { precipitación muy } \\
\text { intensa) }\end{array}$ & $\begin{array}{l}\text { Número de días en un año } \\
\text { en que } P R C P>=20 \mathrm{~mm}\end{array}$ & Días \\
\hline WSDI & $\begin{array}{l}\text { Warm spell duration } \\
\text { indicador } \\
\text { (Indicador de la } \\
\text { duración de periodos } \\
\text { calientes) }\end{array}$ & $\begin{array}{l}\text { Contaje anual de días con } \\
\text { por lo menos } 6 \text { días } \\
\text { consecutivos en } \\
\text { TX>90th percentil }\end{array}$ & Días \\
\hline DTR & $\begin{array}{l}\text { Diurnal temperature } \\
\text { range (Rango diurno } \\
\text { de temperatura) }\end{array}$ & $\begin{array}{l}\text { Diferencia media mensual } \\
\text { entre TX y TN }\end{array}$ & ${ }^{\circ} \mathrm{C}$ \\
\hline SU25 & $\begin{array}{l}\text { Summer days } \\
\text { (Días de verano) }\end{array}$ & $\begin{array}{l}\text { Número de días en un año } \\
\text { cuando } \\
\text { diario) }>25^{\circ} \mathrm{C}\end{array}$ & Días \\
\hline TR20 & $\begin{array}{l}\text { Tropical nights } \\
\text { (Noches tropicales) }\end{array}$ & $\begin{array}{l}\text { Número de días en un año } \\
\text { cuando } \\
\text { diario) }>20^{\circ} \mathrm{C}\end{array}$ & Días \\
\hline TXX & Max Tmax & $\begin{array}{l}\text { Valor mensual máximo de } \\
\text { temperatura máxima diaria }\end{array}$ & ${ }^{\circ} \mathrm{C}$ \\
\hline TXn & Min Tmax & $\begin{array}{l}\text { Valor mensual mínimo de } \\
\text { temperatura máxima diaria }\end{array}$ & ${ }^{\circ} \mathrm{C}$ \\
\hline TNX & Max Tmin & $\begin{array}{l}\text { Valor mensual máximo de } \\
\text { temperatura mínima diaria }\end{array}$ & ${ }^{\circ} \mathrm{C}$ \\
\hline TNn & Min Tmin & $\begin{array}{l}\text { Valor mensual mínimo de } \\
\text { temperatura mínima diaria }\end{array}$ & ${ }^{\circ} \mathrm{C}$ \\
\hline CDD & $\begin{array}{l}\text { Consecutive dry days } \\
\text { (Días secos } \\
\text { consecutivos) }\end{array}$ & $\begin{array}{l}\text { Número máximo de días } \\
\text { consecutivos con } R R<1 \mathrm{~mm}\end{array}$ & Días \\
\hline TN10p & $\begin{array}{l}\text { Cool nights } \\
\text { (Noches frías) }\end{array}$ & $\begin{array}{l}\text { Porcentaje de días cuando } \\
\text { TN }<10 \text { th percentil }\end{array}$ & Días \\
\hline
\end{tabular}




\begin{tabular}{|l|l|l|l|}
\hline TN90p & $\begin{array}{l}\text { Warm nights } \\
\text { (Noches calientes) }\end{array}$ & $\begin{array}{l}\text { Porcentaje de días cuando } \\
\text { TN>90th percentil }\end{array}$ & Días \\
\hline TMAXMEAN & $\begin{array}{l}\text { Temperatura máxima } \\
\text { promedio }\end{array}$ & $\begin{array}{l}\text { Promedio la } \\
\text { temperatura máxima anual }\end{array}$ & ${ }^{\circ} \mathrm{C}$ \\
\hline TMINMEAN & $\begin{array}{l}\text { Temperatura mínima } \\
\text { promedio }\end{array}$ & $\begin{array}{l}\text { Promedio de la } \\
\text { temperatura mínima anual }\end{array}$ & ${ }^{\circ} \mathrm{C}$ \\
\hline PRCPTOT & $\begin{array}{l}\text { Annual total wet-day } \\
\text { precipitation } \\
\text { (Precipitación total } \\
\text { anual en los días } \\
\text { húmedos) }\end{array}$ & $\begin{array}{l}\text { Precipitación anual total en } \\
\text { los } \\
\text { (RR }>=1 \mathrm{~mm} \text { ) }\end{array}$ & $\mathrm{m}$ \\
\hline
\end{tabular}

Fuente: ETCCDI.

Para el cálculo de datos bioclimáticos, de las múltiples metodologías que se han planteado para calcular el índice de aridez, se emplearon los de Martonne, Dantin-Revenga y Gaussen; Así como el uso de diagramas ombrotérmicos o climográmas para determinar cuáles son los periodos de sequía y cuales los de Iluvia en la REBIVI, Mulegé, Baja California Sur.

\section{RESULTADOS Y DISCUSIÓN}

\section{EVALUACIÓN DE HOMOGENEIDAD DE LA SERIE DE DATOS.}

La figura siguiente muestra que las series de tiempo para temperatura máxima (TMAX) y temperatura mínima (TMIN), no son homogéneas, ya que la línea no es recta y presentan saltos o puntos de cambio en el año 1968 y 2001 para TMAX y 1968, 1978,1982 y 2007 para TMIN. En cuanto a la serie de precipitación (PRCP) si es homogénea, ya que la línea es recta y no presenta saltos o puntos de cambio (línea roja).

Estos puntos de cambio no se deben a que los datos de la serie estén erróneos, sino más bien a eventos climatológicos, en el caso de TMAX, con información de la NOAA, el año de 1968 se caracterizó por ser año Niño moderado, en el invierno de ese año (1968), se presentaron anomalías negativas a lo largo de la Península de Baja California.

Los años 1982, 1987, 1998 y 2006 fueron año Niño, o fase cálida, y los años 1971, 2000, 2007 y 2010 se caracterizaron por ser año Niña, o fase fría (Sheinbaum 2003).

Para el caso de TMIN, en el año de 1977 se presentó el fenómeno del Niño débil, durante ese año, en la estación de otoño e invierno se observaron condiciones de normales a por debajo del promedio en el norte y noroeste de México (Figura 3). 


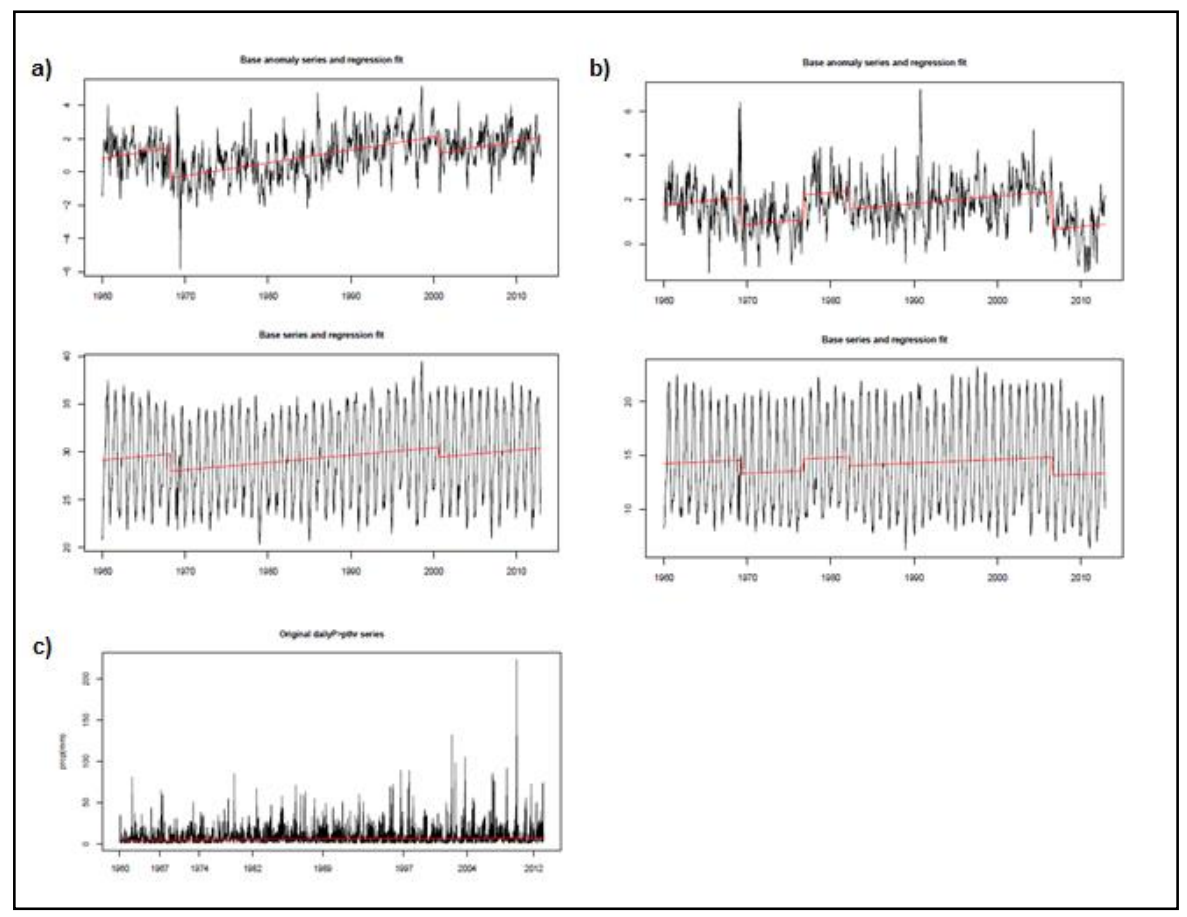

FIGURA 3 - Serie de tiempo de a) temperatura máxima, b) temperatura mínima y c) precipitación en las que se aprecia que las series son homogéneas para la precipitación, ya que no presentan la ocurrencia de saltos o cambios abruptos (línea roja). En cuanto a la temperatura máxima presentan punto de cambio en los años 1968 y 2000, y en la temperatura mínima en los años 1968, 1977, 1982 y 2007, por lo que se considera como no homogéneas. Fuente: Elaboración propia con datos de la CONAGUA, RClimDex, RHtestsV4 y RHtests_dlyPrcp

\section{BIOCLIMATOLOGÍA EN LA RESERVA DE LA BIOSFERA EL VIZCAÍNO, B.C.S.}

El índice aridez propuesto por Gaussen presentó meses lluviosos y meses secos para la Reserva de la Biosfera El Vizcaíno, B.C.S. (REBIVI), la tendencia temporal a lo largo de 50 años muestra a cinco meses lluviosos, siendo los meses de enero, julio, agosto, septiembre y diciembre, el resto fueron meses secos (febrero, marzo, abril, mayo, junio, octubre y noviembre). La precipitación total anual fue de $489.3 \mathrm{~mm}$, y una temperatura media de 21.8 ${ }^{\circ} \mathrm{C}$, considerado de acuerdo a los índices de aridez de Martonne como clima semiárido, y de Dantin-Revenga como zona árida.

De acuerdo a la Secretaría del Medio Ambiente y los Recursos Naturales (SEMARNAT), la REBIVI se considera como Región Ganadera Semiárida, ya que presenta precipitaciones promedio superiores a los $350 \mathrm{~mm}$ al año. Razón por la cual son tierras óptimas para la ganadería bovina, caprina y ovina en forma extensiva y semi-extensiva.

Fueron once los meses que mostraron déficit en la cantidad de lluvia registrada, es decir, que tuvieron una precipitación menor a $100 \mathrm{~mm}$, y solo el mes de agosto mostró superávit en los registros de precipitaciones $(113 \mathrm{~mm})$, por lo que no se considera como una región apta para la agricultura de temporal (Tablas 2 y 3 ). 
TABLA 2 - Precipitación y temperatura media mensual e índices de aridezReserva de la Biosfera El Vizcaíno, Mulegé, B.C.S., 1960-2012.

\section{Índices de aridez}

\begin{tabular}{|c|c|c|c|c|c|c|c|c|}
\hline Mes & $\begin{array}{l}\text { Precipitación } \\
\text { media mensual } \\
(\mathrm{mm})\end{array}$ & \begin{tabular}{|c|} 
Temperatura \\
media mensual \\
$($ tmax+tmin $) / 2$ \\
$\left({ }^{\circ} \mathrm{C}\right)$
\end{tabular} & $\begin{array}{c}\text { Temperatura Media } \\
\text { (suma total temp. } \\
\text { media) } \\
\left({ }^{\circ} \mathrm{C}\right)\end{array}$ & $\begin{array}{c}\text { Total } \\
\text { precipitaciones } \\
(\mathbf{m m})\end{array}$ & Martonne & $\begin{array}{c}\text { Dantin- } \\
\text { Revenga }\end{array}$ & & Gaussen \\
\hline$E$ & 37.1 & 16.1 & 21.80 & 489.3 & 15.39 & 4.45 & $\mathrm{E}$ & Mes lluvioso \\
\hline $\mathrm{F}$ & 32.8 & 16.9 & & & & & $\mathrm{~F}$ & Mes seco \\
\hline$M$ & 17.1 & 18.4 & & & & & M & Mes seco \\
\hline$A$ & 9.2 & 20.1 & & & & & $A$ & Mes seco \\
\hline$M$ & 6.1 & 21.9 & & & & & M & Mes seco \\
\hline 了 & 10.4 & 24.7 & & & & & J & Mes seco \\
\hline J & 64.5 & 27.6 & & & & & J & Mes Iluvioso \\
\hline A & 113.0 & 28.2 & & & & & $A$ & Mes lluvioso \\
\hline $\mathrm{S}$ & 98.5 & 27.2 & & & & & $\mathrm{~S}$ & Mes Iluvioso \\
\hline $\mathrm{O}$ & 32.4 & 23.9 & & & & & $\mathrm{O}$ & Mes seco \\
\hline $\mathrm{N}$ & 27.0 & 19.9 & & & & & $\mathrm{~N}$ & Mes seco \\
\hline D & 41.2 & 16.6 & & & & & $\mathrm{D}$ & Mes lluvioso \\
\hline
\end{tabular}

Fuente: Elaboración propia, con datos de bioclimatología.

TABLA 3 - Precipitación y temperatura media mensual e índices de aridez

\begin{tabular}{|c|c|c|c|c|c|c|}
\hline \multirow{2}{*}{ Mes } & \multirow{2}{*}{$\begin{array}{c}\text { Precipitación } \\
\text { media } \\
\text { mensual } \\
(\mathbf{m m})\end{array}$} & \multirow{2}{*}{$\begin{array}{c}\text { Temperatura } \\
\text { media mensual } \\
(\text { tmax }+ \text { tmin }) / 2 \\
\left({ }^{\circ} \mathrm{C}\right)\end{array}$} & \multirow{2}{*}{ 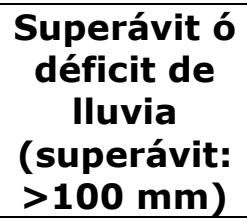 } & \multicolumn{3}{|c|}{ Índices de aridez } \\
\hline & & & & Gaussen & Martonne & $\begin{array}{l}\text { Dantin- } \\
\text { Revenga }\end{array}$ \\
\hline$E$ & 37.1 & 16.1 & Déficit & Mes Iluvioso & & \\
\hline$F$ & 32.8 & 16.9 & Déficit & Mes seco & 15.39 & 4.45 \\
\hline$M$ & 17.1 & 18.4 & Déficit & Mes seco & & \\
\hline$A$ & 9.2 & 20.1 & Déficit & Mes seco & & $\frac{\pi}{\pi} \pi$ \\
\hline M & 6.1 & 21.9 & Déficit & Mes seco & 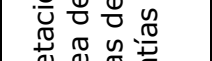 & 贾 \\
\hline J & 10.4 & 24.7 & Déficit & Mes seco & 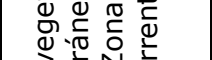 & 范 \\
\hline J & 64.5 & 27.6 & Déficit & Mes Iluvioso & 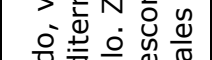 & :음 $>\overline{0}$ \\
\hline$A$ & 113.0 & 28.2 & Superávit & Mes Iluvioso & 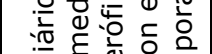 & 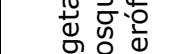 \\
\hline $\mathrm{S}$ & 98.5 & 27.2 & Déficit & Mes Iluvioso & 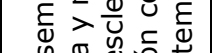 & $>0 \bar{y}$ \\
\hline $\mathrm{O}$ & 32.4 & 23.9 & Déficit & Mes seco & 足 & \\
\hline $\mathrm{N}$ & 27.0 & 19.9 & Déficit & Mes seco & 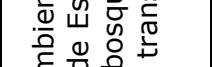 & 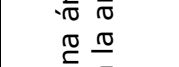 \\
\hline $\mathrm{D}$ & 41.2 & 16.6 & Déficit & Mes lluvioso & & 두 \\
\hline
\end{tabular}

Fuente: Elaboración propia, con datos de bioclimatología. 
A lo largo de cinco décadas, el mes de agosto fue el que promedió la mayor cantidad de lluvia, al registrar una precipitación de $113.0 \mathrm{~mm}$, y el período con menor cantidad registrada fue el mes de mayo, con tan solo 6.1 $\mathrm{mm}$.

En cuanto a la distribución de las precipitaciones a lo largo del año, estas fueron muy irregulares, ya que se presentaron 11 meses secos.

De acuerdo al índice de Gaussen, se considera como periodo o mes seco cuando las precipitaciones son menores que el doble de la temperatura (2T>P), y periodo lluvioso cuando las precipitaciones son mayores que el doble de la temperatura $(2 \mathrm{~T}<\mathrm{P})$, en la figura siguiente se muestra el diagrama ombrotérmico (o de Gaussen-Walter), donde el área en color amarillo representa los meses de sequía y el área azul los meses lluviosos.

Los meses con índice de aridez o meses de sequía fueron de febrero a junio, siendo el mes de mayo el periodo con mayor grado de sequía.

En el mes de enero se registró la temperatura media más fría al promediar $16.1{ }^{\circ} \mathrm{C}$ y el mes con la temperatura más cálida fue agosto, alcanzando los $28.2^{\circ} \mathrm{C}$.

Al calcular la diferencia de temperaturas entre estos dos meses, el intervalo resultante fue de $12.1{ }^{\circ} \mathrm{C}$, clasificándose como una amplitud térmica media (Figura 4). 

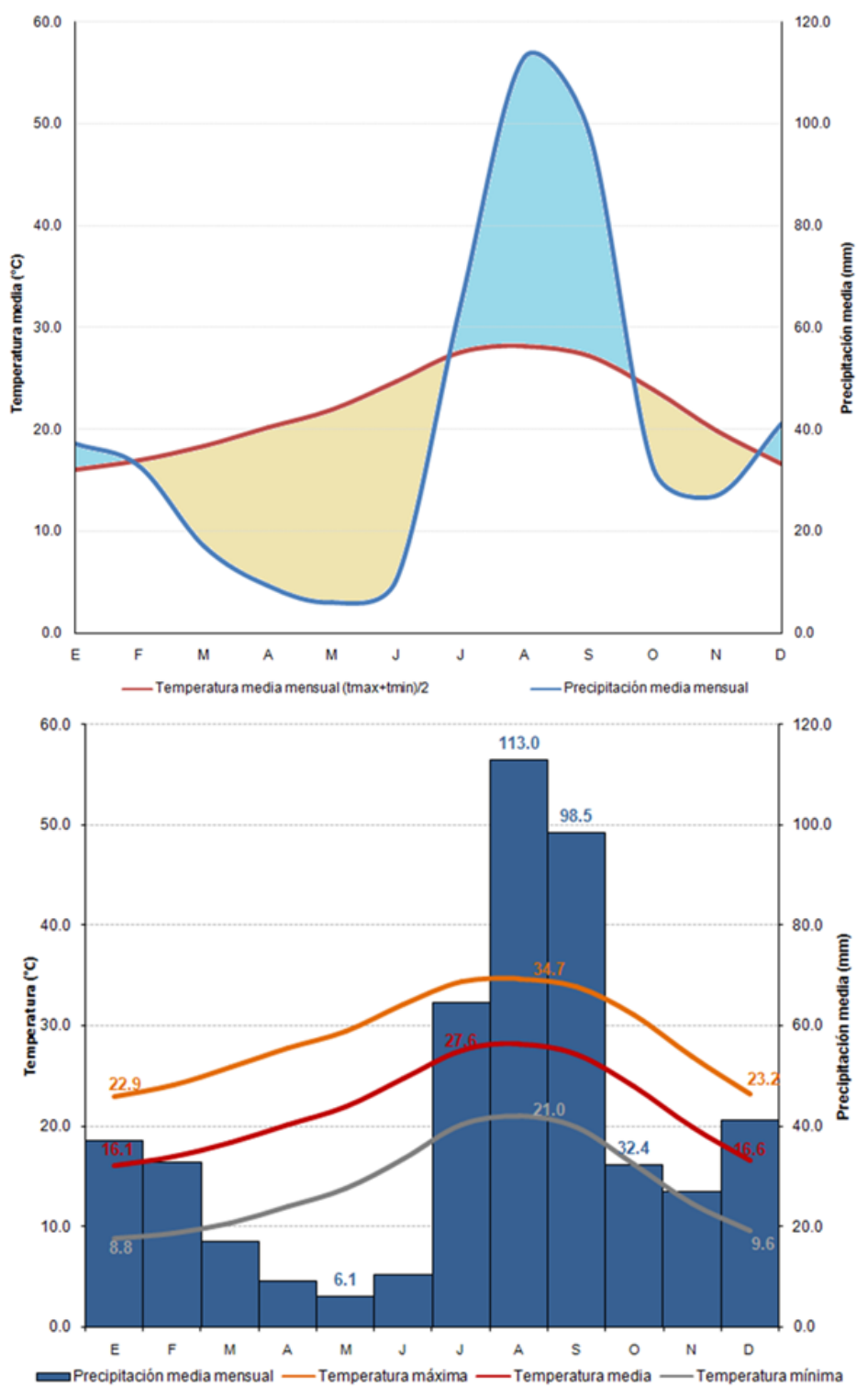

FIGURA 4 - Diagrama ombrotérmico (climograma) de la REBIVI, B.C.S., 1960-2012. Figura de arriba. Área color amarillo: Periodo de aridez, área color azul: Periodo de lluvia

\section{RESULTADOS DE LOS ÍNDICES DE CAMBIO CLIMÁTICO OBTENIDOS}

La tendencia mostrada a lo largo de cincuenta años es de aumento en el máximo número en 1 año de días consecutivos con precipitación mayor a $1 \mathrm{~mm}$, así tenemos que en 1960 era de 4 días consecutivos al año con precipitaciones mayores a $1 \mathrm{~mm}$, y para el 2012 pasó a 5 días consecutivos al año con precipitaciones mayores a $1 \mathrm{~mm}$, el máximo fue en los años 1993 y 1997, influenciado principalmente por la presencia del fenómeno El Niño, en los años 1993, 1996 y 1997, donde se registraron 11 días con lluvias mayores a 1mm, y el mínimo de días fue en 1968, 1979 y 2011, con 3 días respectivamente. 
En cinco décadas, aunque la tendencia sea moderada, han aumentado el número de días por año que han registrado precipitaciones mayores a $1 \mathrm{~mm}$. (Figura 5).

\section{CWD REBIVI}

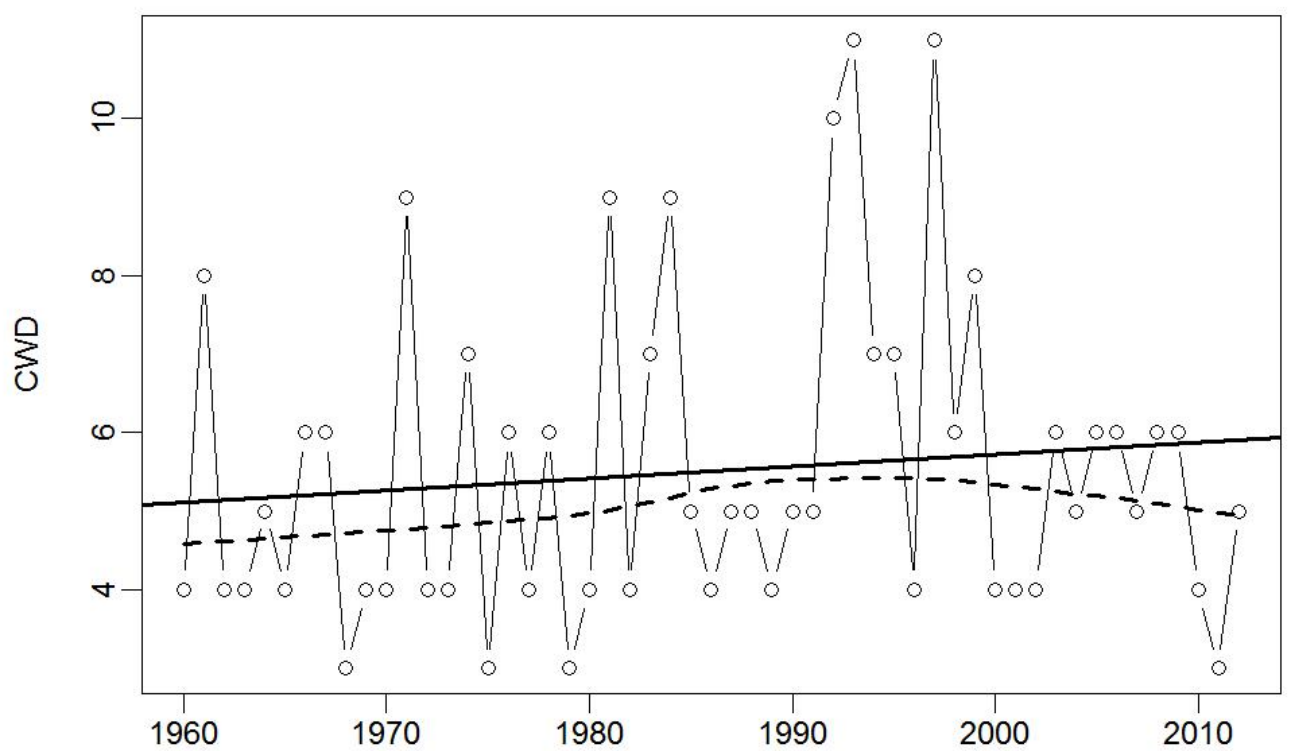

FIGURA 5 - Longitud máxima de la racha lluviosa (CWD), 1960-2012. Fuente: Elaboración propia en RClimDex, con datos de CONAGUA

Las evidencias más relevantes de las tendencias en los índices del número anual de días con lluvias fuertes, muestran que el número anual de días en que la precipitación es mayor a $20 \mathrm{~mm}$ ha sido de incremento, en el año de 1960 era de 2 días con precipitación mayor a 20 mm en un año, pasó en el 2012 a 11 días con precipitaciones mayores a $20 \mathrm{~mm}$, el máximo fue en el año 1984, con 17 días y el mínimo en 1968, donde no se registró un solo día con precipitación mayor a $20 \mathrm{~mm}$. La tendencia de incremento a lo largo de 5 décadas pudiera deberse a la presencia cada vez mas de eventos meteorológicos, como huracanes o tormentas tropicales, o a patrones climáticos recurrentes, como el fenómeno de El Niño(Figura 6). 


\section{R20MM REBIVI}

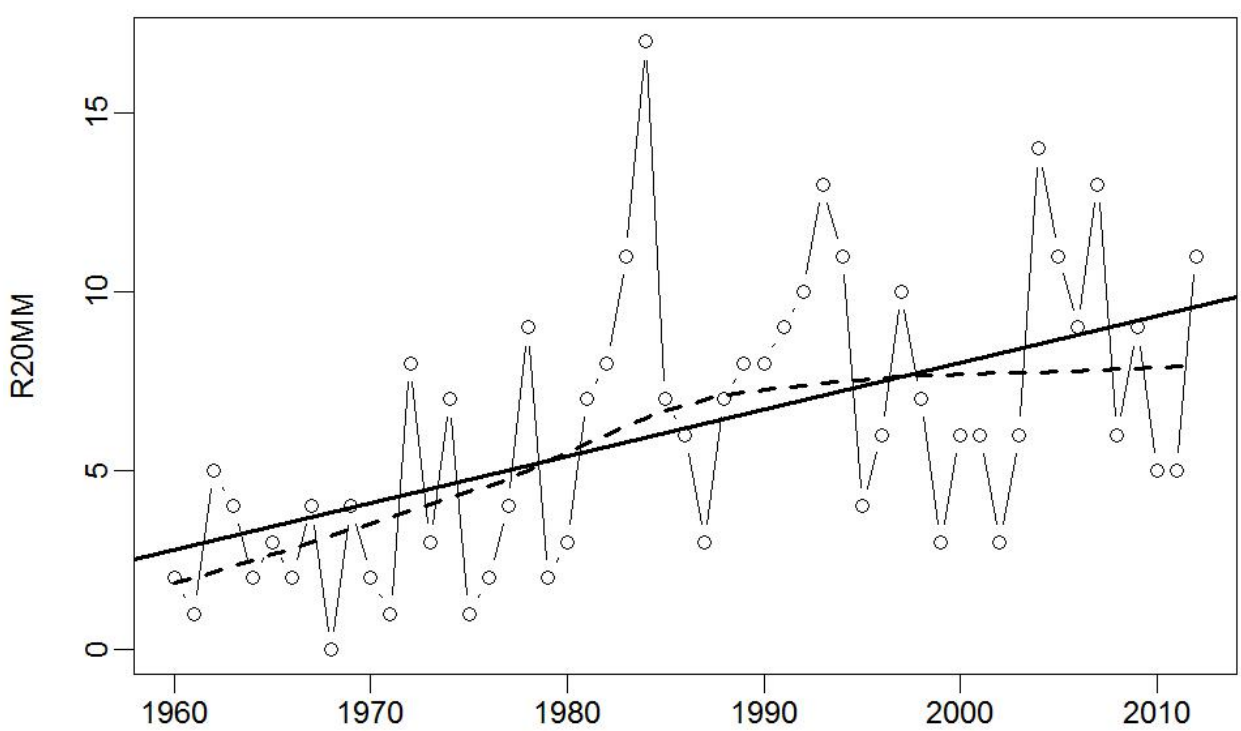

FIGURA 6 - Días de precipitación abundante (R20MM), 1960-2012. Fuente: Elaboración propia en RClimDex, con datos de CONAGUA

La tendencia mostrada es de aumento en el número de días consecutivos con periodos calientes al año, en 1960 era de 20 días consecutivos con periodos calientes, en el año 2012 no presentó días consecutivos con periodos calientes, el máximo fue en el año 1998, al registrar 56 días consecutivos. Se ha producido un moderado incremento en la temperatura, al aumentar el número de días consecutivos con periodos de calientes, tal como lo muestra la figura siguiente (Figura 7).

\section{WSDI REBIVI}

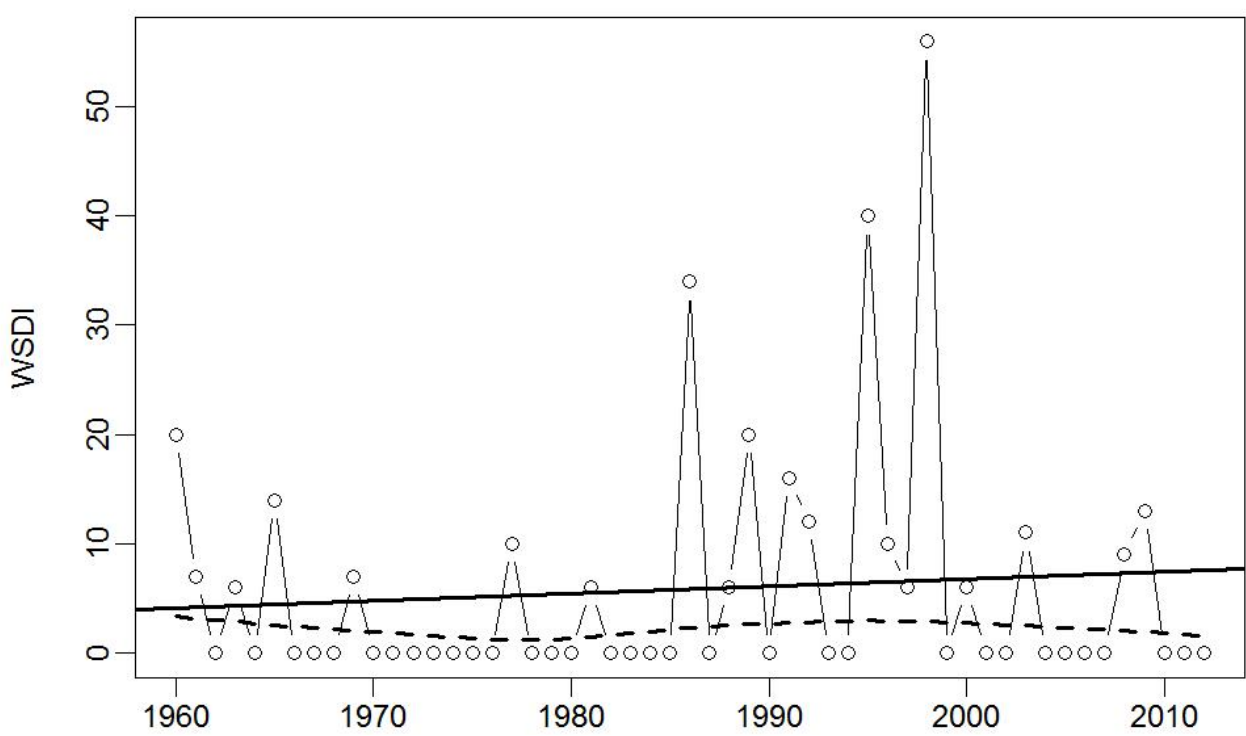

FIGURA 7 - Indicador de duración de racha cálida (WSDI), 1960-2012.Fuente: Elaboración propia en RClimDex, con datos de CONAGUA 
El rango diurno de temperatura (Figura 8), es decir, la diferencia entre la temperatura máxima y la temperatura mínima, ha ido en aumento en cincuenta años en la REBIVI, al pasar de $14.8^{\circ} \mathrm{C}$ en 1960 a $16^{\circ} \mathrm{C}$ en el año 2012 , el máximo fue en el año 2010 , con $17.62^{\circ} \mathrm{C}$ y el mínimo en 1978 , con $13.15^{\circ} \mathrm{C}$. Se ha incrementado el rango entre ambas temperaturas, es decir, la diferencia entre temperatura máxima y temperatura mínima, la explicación se debe a que la temperatura máxima promedio se ha incrementado, como lo muestra la figura 18, y por otro lado, la temperatura mínima promedio ha ido en decremento, mostrado en la Figura 19.

\section{DTR REBIVI}

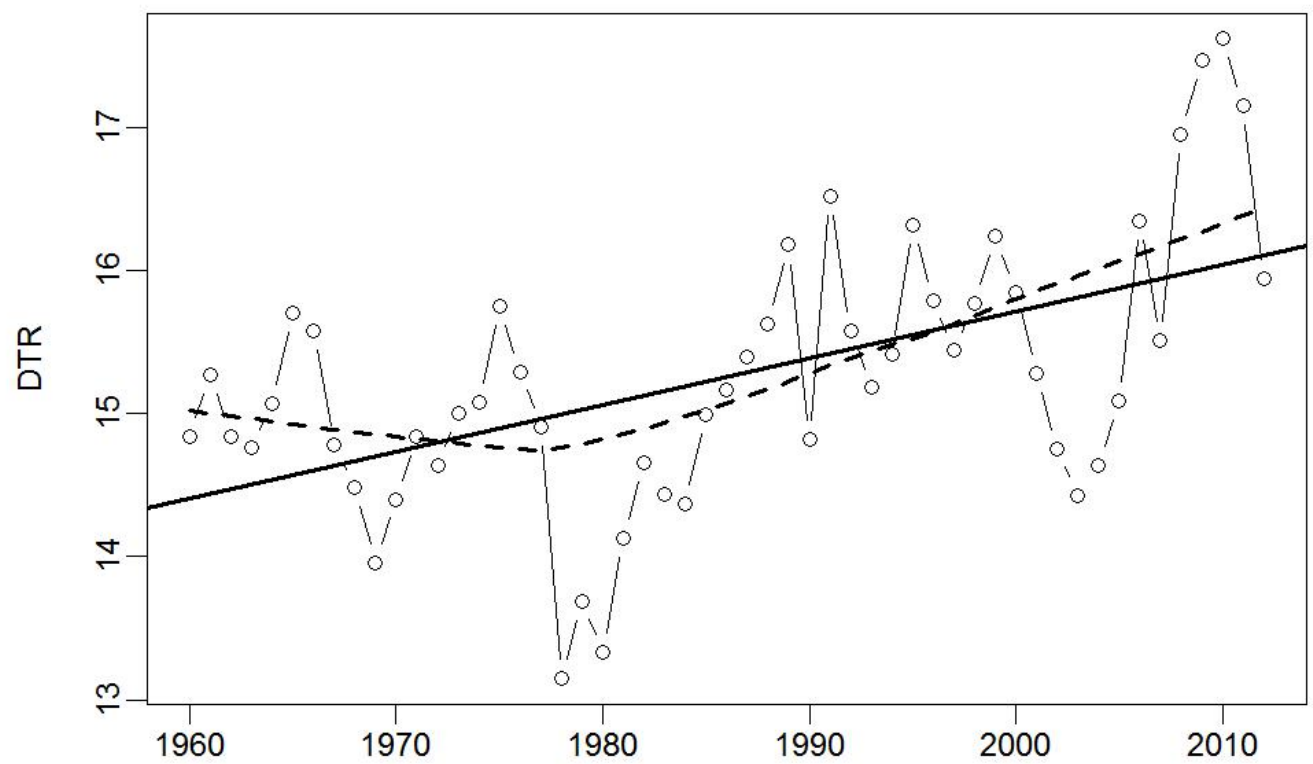

FIGURA 8 - Rango diurno de temperatura (DTR), 1960-2012. Fuente: Elaboración propia en RClimDex, con datos de CONAGUA

También el número de días de verano donde la temperatura máxima es mayor a $25^{\circ} \mathrm{C}$ se ha incrementado, en 1960 era de 270 días de verano al año, y para el año 2012 aumentó a 273 días de verano con temperaturas superiores a los $25^{\circ} \mathrm{C}$, el máximo fue en el año 1996, con 316 días y el mínimo en los años 1964 y 1978, con 241 días respectivamente. Se ha registrado un aumento en el número de días por año en donde la temperatura ha sido mayor a los $25^{\circ} \mathrm{C}$. (Figura 9). 


\section{SU25 REBIVI}

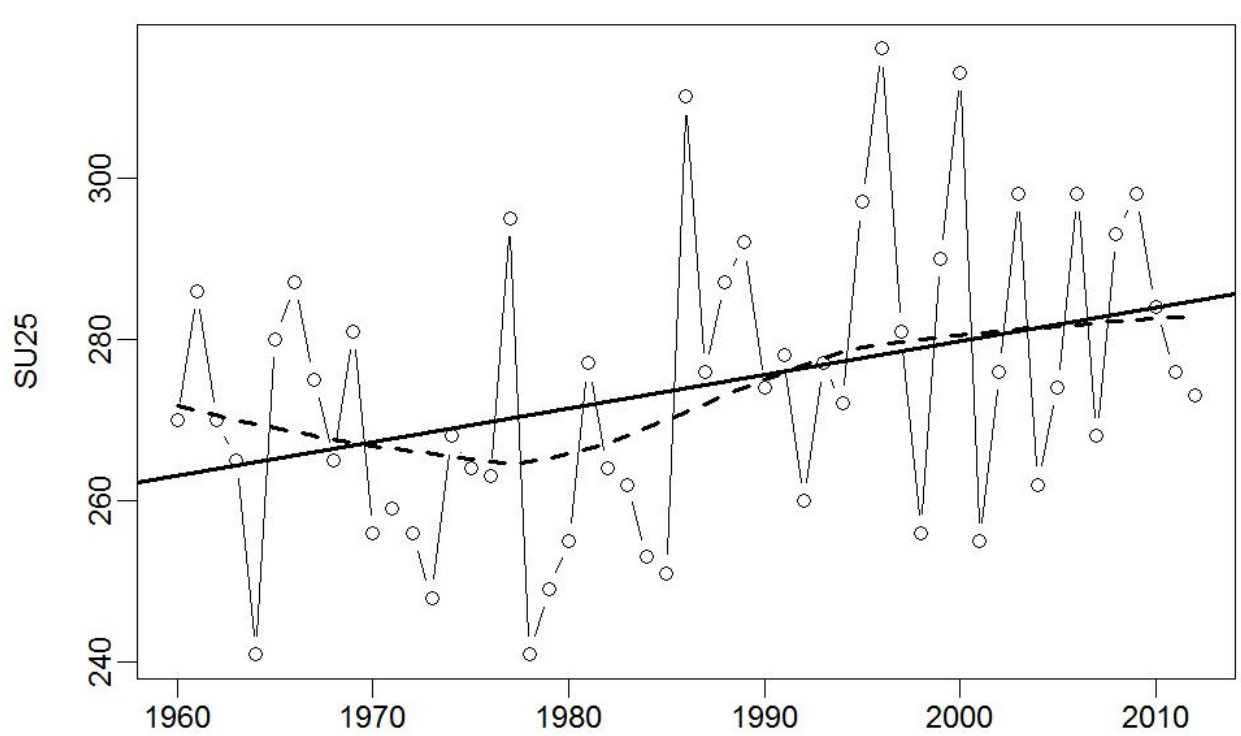

FIGURA 9 - Días de verano (SU25), 1960-2012. Fuente: Elaboración propia en RClimDex, con datos de CONAGUA

La tendencia a lo largo de cinco décadas es de aumento en el número de noches tropicales donde la temperatura mínima es mayor a $20{ }^{\circ} \mathrm{C}$, la gráfica muestra que en el año 1960 era de 79 noches tropicales y para el año 2012 fue de 35 noches tropicales donde la temperatura mínima era mayor a $20{ }^{\circ} \mathrm{C}$, el máximo fue en 1997, con 95 noches tropicales y el mínimo en los años 2008 y 2010, con 1 noche tropical ( $>20^{\circ} \mathrm{C}$ ) (Figura 10).

\section{TR20 REBIVI}

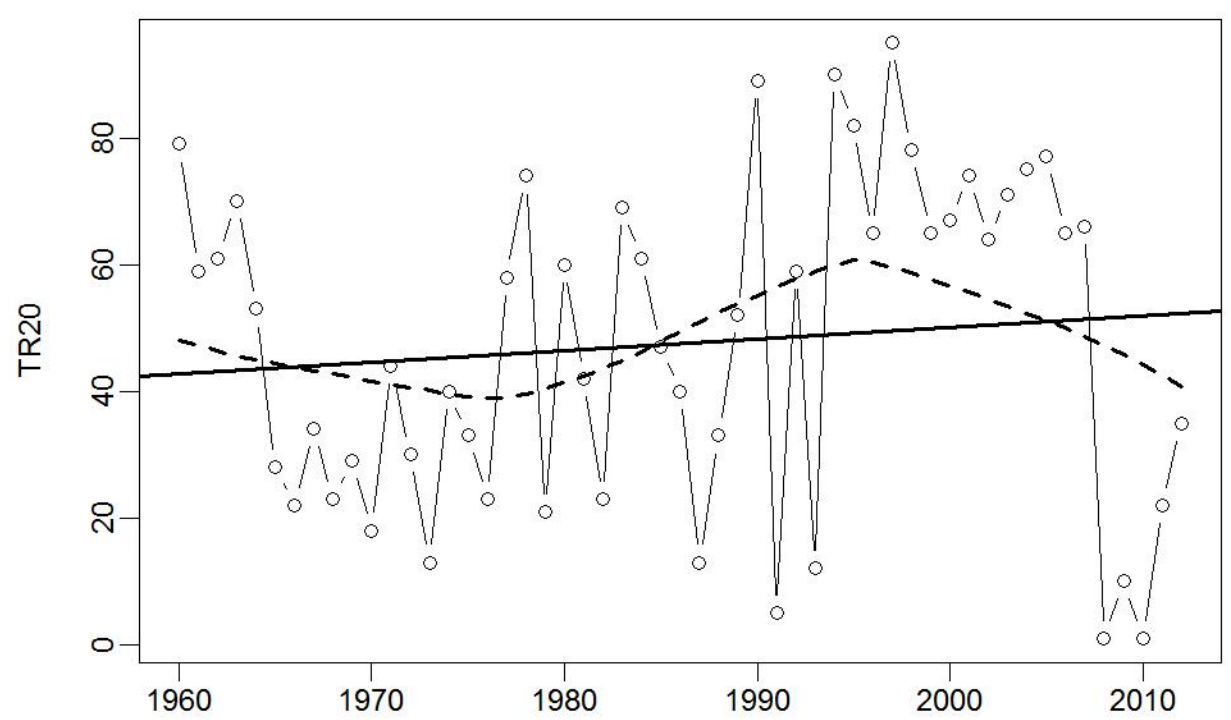

FIGURA 10 - Noches tropicales (TR20), 1960-2012. Fuente: Elaboración propia en RClimDex, con datos de CONAGUA. 
La tendencia en la temperatura máxima extrema es visiblemente de aumento, en 1960 la temperatura máxima más alta mensual fue de $39{ }^{\circ} \mathrm{C}$ y para el año 2012 pasó a ser de $38{ }^{\circ} \mathrm{C}$, el máximo fue en los años 1998 y 2009, con $41^{\circ} \mathrm{C}$ respectivamente, y el mínimo en 1970 , con $35^{\circ} \mathrm{C}$. Éste aumento en la tendencia de la temperatura máxima extrema puede provocar periodos prolongados de sequías. Se presume que en la actualidad hay mayores sequías que antaño, muestra de los efectos del cambio climático. Esta situación, traducida en desastre natural, implica un desequilibrio hidrológico y los abastecimientos de agua presentan niveles inferiores a los normales, provocando trastornos socioeconómicos. (Figura 11).

\section{TXX REBIVI}

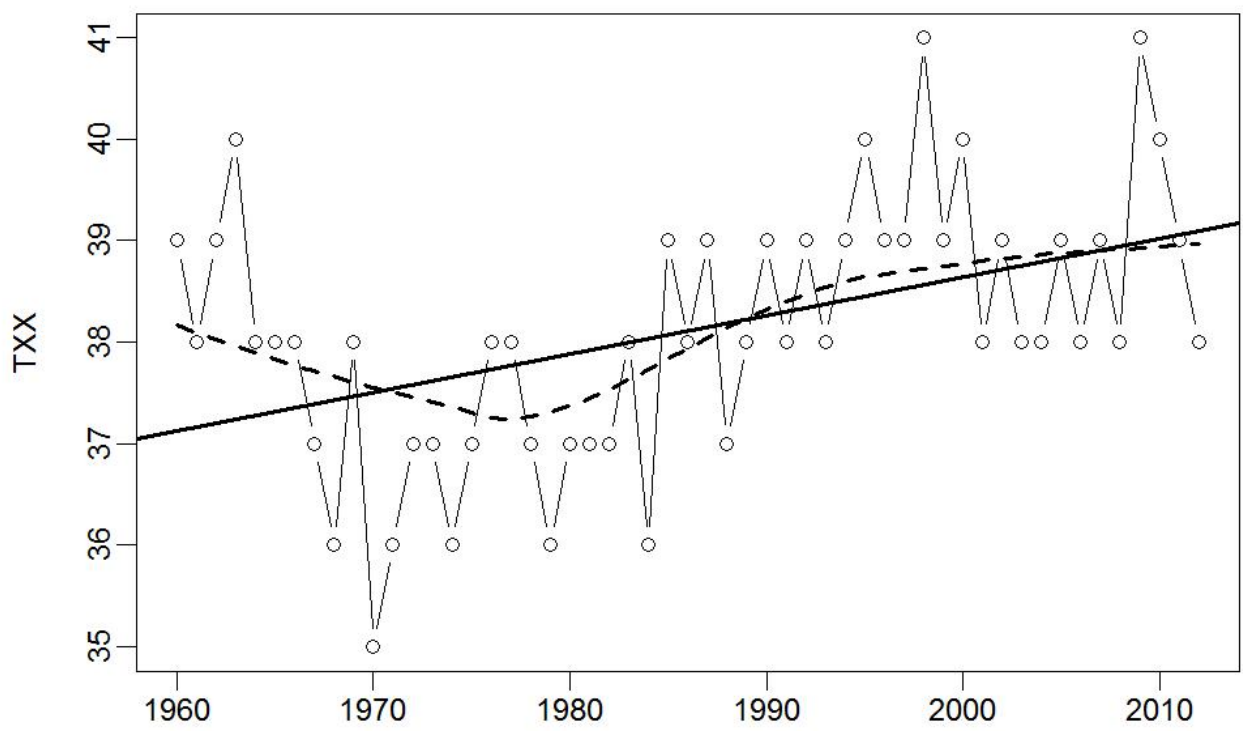

FIGURA 11 - Temperatura máxima extrema (TXX), 1960-2012. Fuente: Elaboración propia en RClimDex, con datos de CONAGUA

En lo que respecta a la tendencia en la temperatura máxima más baja, también muestra un incremento, siendo de $16^{\circ} \mathrm{C}$ en 1960 y de $19^{\circ} \mathrm{C}$ en el año 2012, el máximo fue en el año 2000 , al registrarse $23^{\circ} \mathrm{C}$ en la temperatura máxima más baja. Esto significa que la temperatura máxima más baja ha presentado aumento en grados centígrados en relación a años anteriores, dicho en otras palabras, refleja un aumento de calor (Figura 12). 
TXN REBIVI

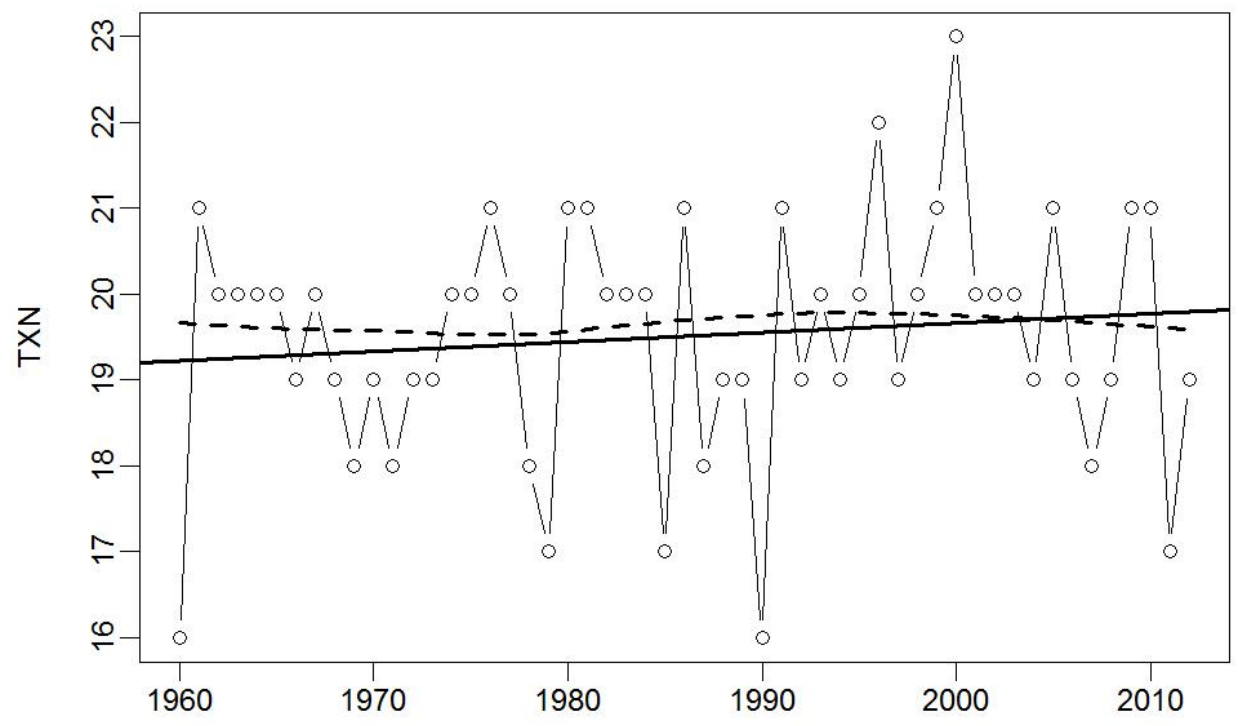

Figura 12 - Temperatura máxima más baja (TXN), 1960-2012. Fuente: Elaboración propia en RClimDex, con datos de CONAGUA

La tendencia es de un ligero aumento en la temperatura mínima más alta, esta era de $24^{\circ} \mathrm{C}$ en el año 1960 , y de $22^{\circ} \mathrm{C}$ en el año 2012 , el máximo fue en los años 1978,1995 y 1997 , con $25^{\circ} \mathrm{C}$ en cada uno de estos años, y el mínimo en los años 1979, 1991, 1993, 2008 y 2010, con $21{ }^{\circ} \mathrm{C}$ respectivamente. También la temperatura mínima más alta presenta un leve aumento en los niveles de calor a través de cinco décadas (Figura 13).

TNX REBIVI

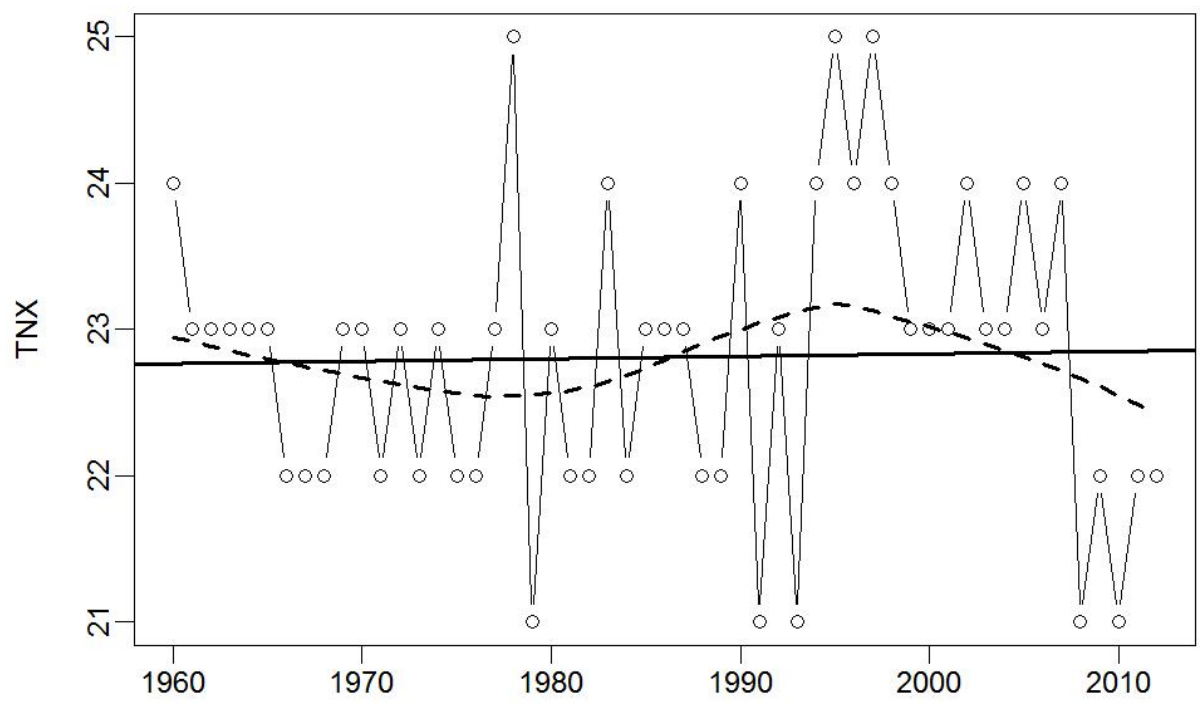

FIGURA 13 - Temperatura mínima más alta (TNX), 1960-2012. Fuente: Elaboración propia en RClimDex, con datos de CONAGUA 
La temperatura mínima más baja presentó una tendencia de disminución, en el año de 1960 era de $5^{\circ} \mathrm{C}$, y pasó a los $6^{\circ} \mathrm{C}$ en el año 2012, el máximo fue en los años $1961,1980,1981$, y 1993 , con $8^{\circ} \mathrm{C}$ en cada uno de estos años, y el mínimo en los años 1985, 1989, 2000 y 2006, con $4{ }^{\circ} \mathrm{C}$ respectivamente. Esto significa que en cinco décadas se ha presentado una tendencia de descenso en los registros de la temperatura mínima (Figura 14).

TNN REBIVI

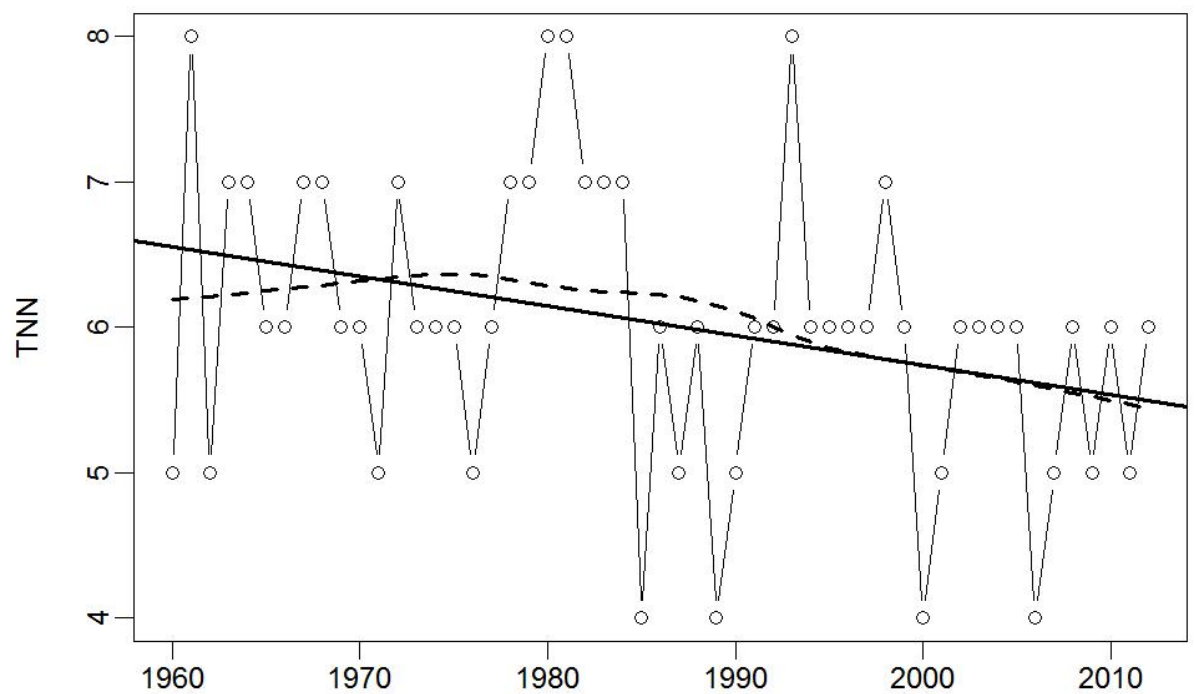

Figura 14 - Temperatura mínima extrema (TNN), 1960-2012. Fuente: Elaboración propia en RClimDex, con datos de CONAGUA

El número consecutivo de días sin lluvia ha mostrado un decremento en la tendencia, pasó de 112 días consecutivos en el año 1960 a 115 días consecutivos sin Iluvia en el 2012, el máximo fue en el año 1967, con 215 días y el mínimo en 1992, con 53 días, el máximo fue en los años 1993 y 1997, con 11 días y el mínimo en 1968, 1979 y 2011, con 3 días respectivamente. Cada vez han ido en disminución el número de días por año que presentan días consecutivos sin presentar precipitaciones, esto influenciado por el incremento en la precipitación (PRCPTOT) a lo largo de los años en esa región (Figura 15). 


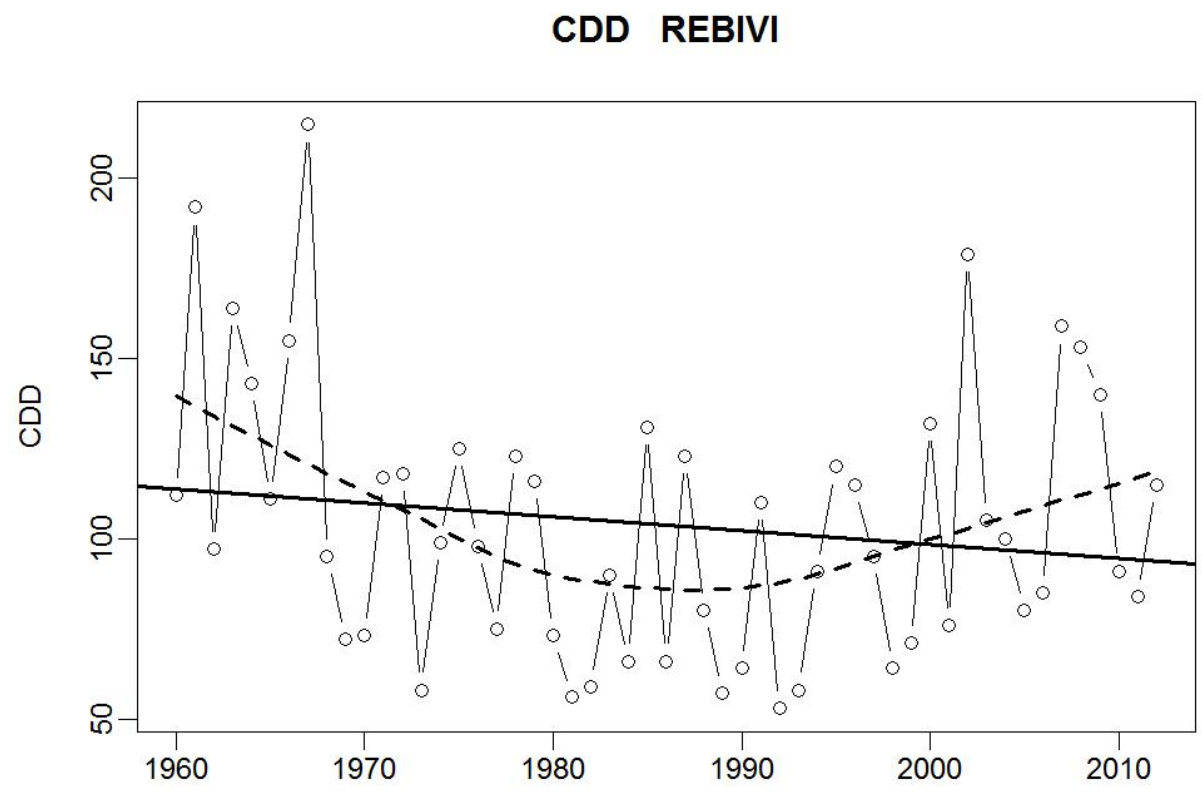

FIGURA 15 - Días consecutivos sin Iluvia (CDD), 1960-2012. Fuente: Elaboración propia en RClimDex, con datos de CONAGUA

Los datos obtenidos en el presente trabajo no concuerdan con lo expuesto por Alexander et al. En el año 2006, de acuerdo a que según reportes en los últimos 50 años ha disminuido el número de noches frías y el número de noches cálidas han ido en aumento en $70 \%$ del planeta, ya que esto se ha dado de manera inversa en la REBIVI, es decir, se han incrementado las noches frías y disminuido las noches cálidas (Figuras 16 y 17).

La tendencia en las noches frías es de aumento, en el año de 1960 esta era del $5.73 \%$ y del $2.09 \%$ en el año 2012, el máximo fue en el 2010, con el $28.27 \%$ y el mínimo en 1963 y 2003, donde no se registraron noches frías (Figura 16). 


\section{TN10P REBIVI}

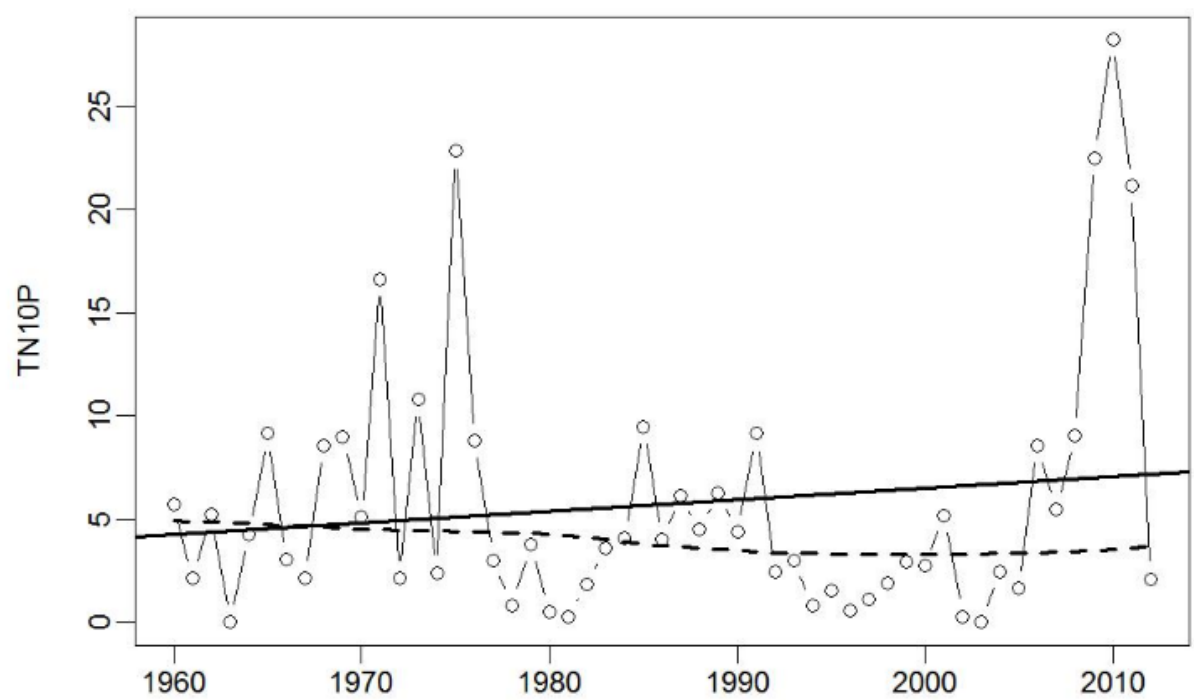

FIGURA 16 - Noches frías (TN10P), 1960-2012. Fuente: Elaboración propia en RClimDex, con datos de CONAGUA

En cincuenta años, se presentó una disminución en el porcentaje de noches cálidas, al pasar del $9.22 \%$ en 1960 a un $1.82 \%$ en el año 2012 , el máximo fue en el año 1990 , con el $26.07 \%$ con noches cálidas y el mínimo en 2008, 2009, 2010 y 2011, donde no se registraron noches cálidas. La tendencia presentada es de una ligera disminución en las noches cálidas (Figura 17).

TN90P REBIVI

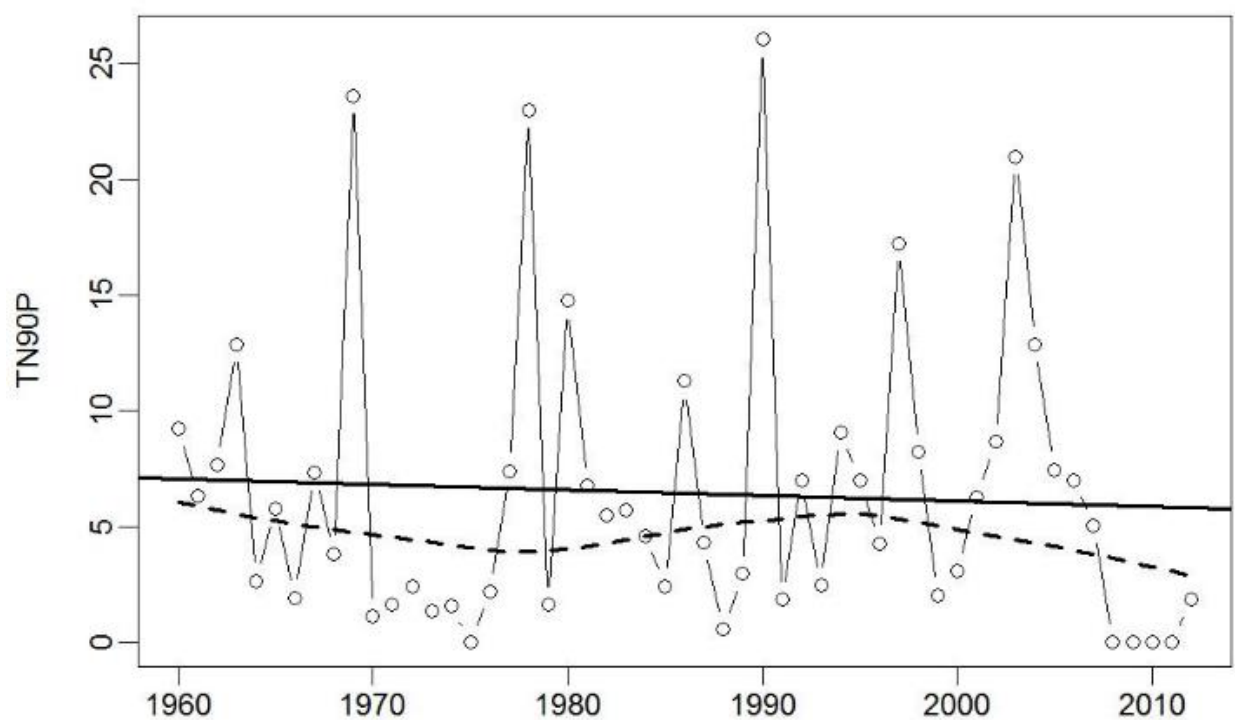

FIGURA 17 - Noches cálidas (TN90P), 1960-2012. Fuente: Elaboración propia en RClimDex, con datos de CONAGUA. 
Los registros muestran que el promedio de la temperatura máxima muestra una clara tendencia de aumento, ya que en el año 1960 era de 29.45 ${ }^{\circ} \mathrm{C}$, llegando a aumentar a los $30.03^{\circ} \mathrm{C}$ en el año 2012 , el máximo fue en el año 1995 , con $30.88^{\circ} \mathrm{C}$, y el mínimo en 1979 , con $27.68^{\circ} \mathrm{C}$ (Figura 18 ).

\section{TMAXMEAN REBIVI}

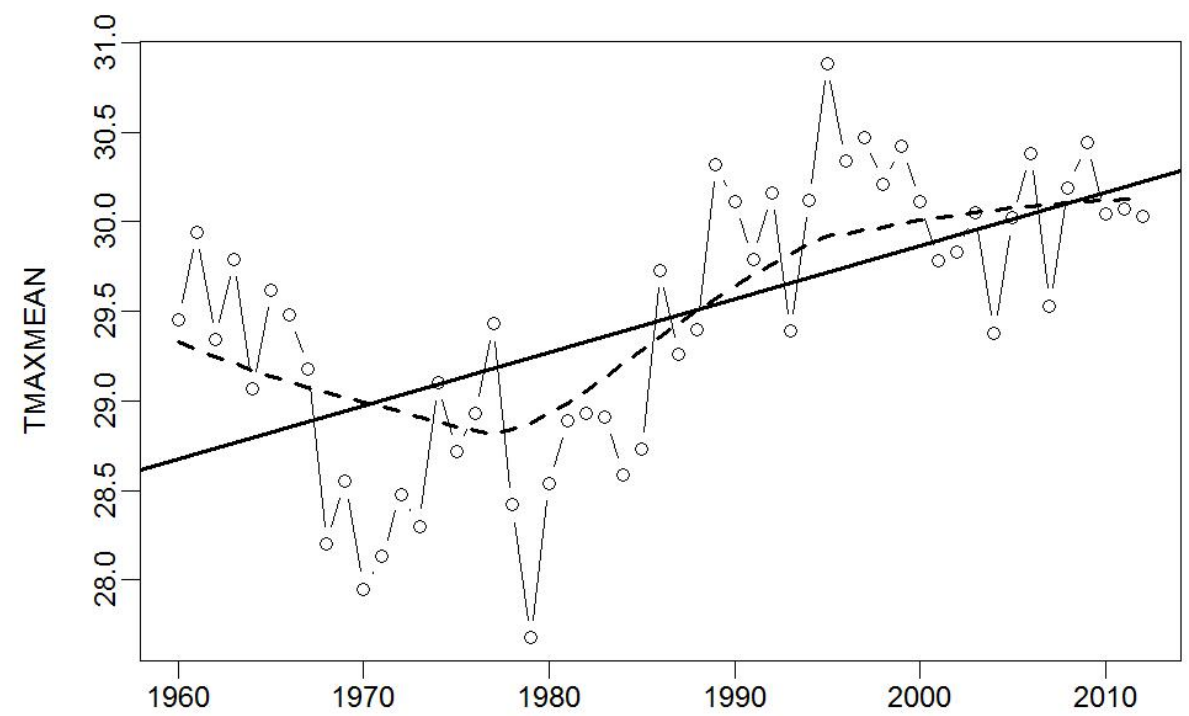

FIGURA 18 - Temperatura máxima promedio (TMAXMEAN), 1960-2012. Fuente: Elaboración propia en RClimDex, con datos de CONAGUA

La temperatura mínima promedio muestra una ligera tendencia de disminución, al ser esta en 1960 de $14.61^{\circ} \mathrm{C}$ a descender a los $14.09^{\circ} \mathrm{C}$ en el año 2012, el máximo reportado fue en el 2003, al registrar $15.61{ }^{\circ} \mathrm{C}$, y el mínimo en el año 2010 , con $12.42^{\circ} \mathrm{C}$. (Figura 19).

\section{TMINMEAN REBIVI}

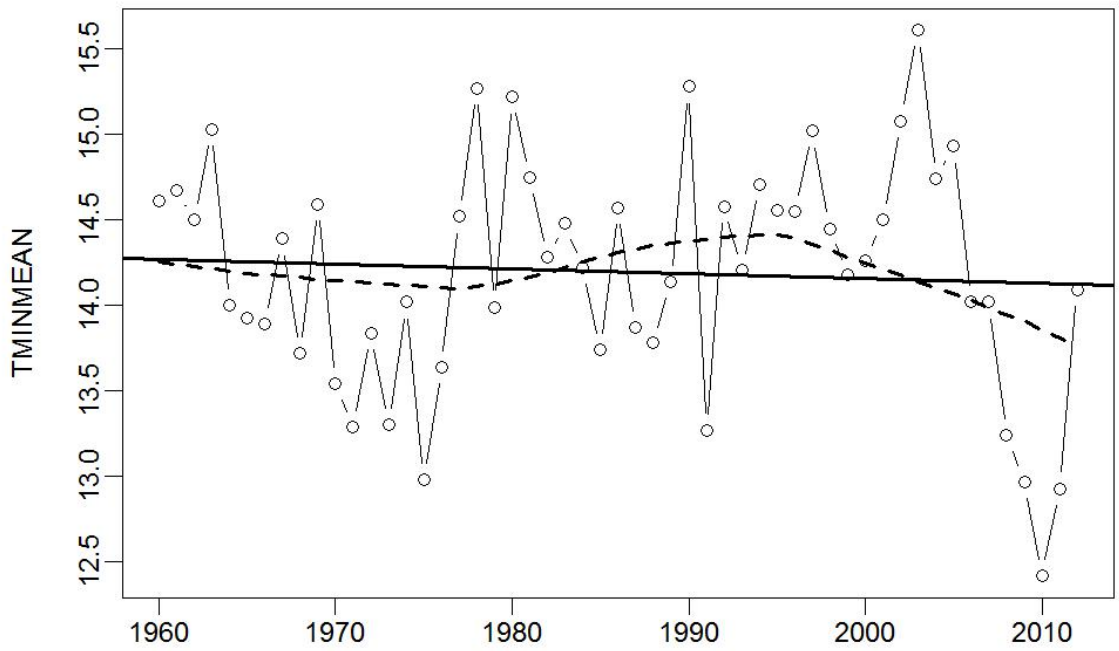

FIGURA 19 - Temperatura mínima promedio (TMINMEAN), 1960-2012.Fuente: Elaboración propia en RClimDex, con datos de CONAGUA 
La tendencia muestra un evidente incremento en la precipitación anual, pasando de $164 \mathrm{~mm}$ en el año 1960, a $716 \mathrm{~mm}$ de precipitación total en el año 2012, el máximo de precipitación fue el registrado en el año 1984, alcanzando los 850 mm, y el mínimo en 1968, donde las lluvias registraron tan solo $57 \mathrm{~mm}$. Las precipitaciones son escasas ya que no se cuenta con lluvias de temporal, la mayor cantidad de agua se obtiene a raíz de eventos hidrometeorológicos como huracanes y tormentas tropicales, una media dentro de las Normas Climatológicas oscila entre $43 \mathrm{~mm}$ y $385 \mathrm{~mm}$ anuales. (Figura 20).

\section{PRCPTOT REBIVI}

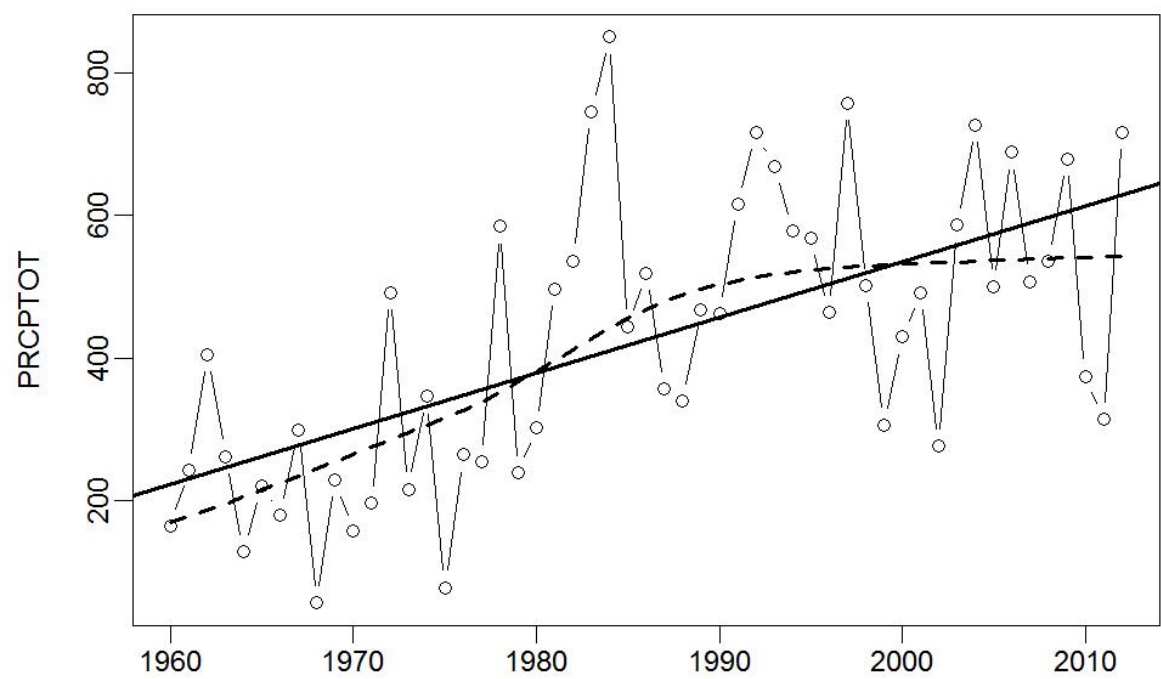

FIGURA 20 - Precipitación total (PRCPTOT), 1960-2012. Fuente: Elaboración propia en RClimDex, con datos de CONAGUA

En poco más de cincuenta años, la temperatura mínima promedio presentó una tasa de crecimiento negativa del $0.07 \%$, mientras que la temperatura máxima promedio tubo una tasa de crecimiento del $0.04 \%$ y la precipitación total un crecimiento cercano al $3 \%$ (2.87 \%) (Tabla 4).

TABLA 4 - Temperatura mínima promedio, temperatura máxima promedio, precipitación total en la Reserva de la Biosfera El Vizcaíno, B.C.S., 1960-2012

\begin{tabular}{|c|c|c|c|}
\hline Año & $\begin{array}{c}\text { TMINMEAN } \\
\left({ }^{\circ} \mathbf{C}\right)\end{array}$ & $\begin{array}{c}\text { TMAXMEAN } \\
\left({ }^{\circ} \mathbf{C}\right)\end{array}$ & $\begin{array}{c}\text { PRCPTOT } \\
(\mathbf{m m})\end{array}$ \\
\hline 1960 & 14.61 & 29.45 & 164 \\
\hline 1961 & 14.67 & 29.94 & 243 \\
\hline 1962 & 14.50 & 29.34 & 405 \\
\hline 1963 & 15.03 & 29.79 & 262 \\
\hline 1964 & 14.00 & 29.07 & 129 \\
\hline 1965 & 13.93 & 29.62 & 221 \\
\hline 1966 & 13.89 & 29.48 & 179 \\
\hline
\end{tabular}




\begin{tabular}{|c|c|c|c|}
\hline 1967 & 14.39 & 29.18 & 299 \\
\hline 1968 & 13.72 & 28.20 & 57 \\
\hline 1969 & 14.59 & 28.55 & 229 \\
\hline 1970 & 13.54 & 27.95 & 157 \\
\hline 1971 & 13.29 & 28.13 & 196 \\
\hline 1972 & 13.84 & 28.48 & 491 \\
\hline 1973 & 13.30 & 28.30 & 216 \\
\hline 1974 & 14.02 & 29.10 & 346 \\
\hline 1975 & 12.98 & 28.72 & 78 \\
\hline 1976 & 13.64 & 28.93 & 264 \\
\hline 1977 & 14.52 & 29.43 & 254 \\
\hline 1978 & 15.27 & 28.42 & 584 \\
\hline 1979 & 13.99 & 27.68 & 239 \\
\hline 1980 & 15.22 & 28.54 & 302 \\
\hline 1981 & 14.75 & 28.89 & 497 \\
\hline 1982 & 14.28 & 28.93 & 536 \\
\hline 1983 & 14.48 & 28.91 & 745 \\
\hline 1984 & 14.22 & 28.59 & 850 \\
\hline 1985 & 13.74 & 28.73 & 444 \\
\hline 1986 & 14.57 & 29.73 & 519 \\
\hline 1987 & 13.87 & 29.26 & 357 \\
\hline 1988 & 13.78 & 29.40 & 339 \\
\hline 1989 & 14.14 & 30.32 & 468 \\
\hline 1990 & 15.28 & 30.11 & 462 \\
\hline 1991 & 13.27 & 29.79 & 616 \\
\hline 1992 & 14.58 & 30.16 & 716 \\
\hline 1993 & 14.21 & 29.39 & 668 \\
\hline 1994 & 14.71 & 30.12 & 578 \\
\hline 1995 & 14.56 & 30.88 & 568 \\
\hline 1996 & 14.55 & 30.34 & 464 \\
\hline 1997 & 15.02 & 30.47 & 757 \\
\hline 1998 & 14.45 & 30.21 & 501 \\
\hline 1999 & 14.18 & 30.42 & 306 \\
\hline 2000 & 14.26 & 30.11 & 429 \\
\hline 2001 & 14.50 & 29.78 & 491 \\
\hline 2002 & 15.08 & 29.83 & 276 \\
\hline 2003 & 15.61 & 30.05 & 586 \\
\hline 2004 & 14.74 & 29.38 & 726 \\
\hline 2005 & 14.93 & 30.02 & 500 \\
\hline 2006 & 14.02 & 30.38 & 688 \\
\hline
\end{tabular}




\begin{tabular}{|c|c|c|c|}
\hline 2007 & 14.02 & 29.53 & 507 \\
\hline 2008 & 13.24 & 30.19 & 535 \\
\hline 2009 & 12.97 & 30.44 & 679 \\
\hline 2010 & 12.42 & 30.04 & 374 \\
\hline 2011 & 12.93 & 30.07 & 314 \\
\hline 2012 & 14.09 & 30.03 & 716 \\
\hline TCPA(\%) & $\mathbf{- 0 . 0 7}$ & $\mathbf{0 . 0 4}$ & $\mathbf{2 . 8 7}$ \\
\hline
\end{tabular}

La figura siguiente muestra la tendencia en las últimas cinco décadas en la REBIVI, donde la temperatura máxima promedio es de incremento, la temperatura mínima promedio es ligeramente de disminución y la precipitación total muestra una clara tendencia de aumento.

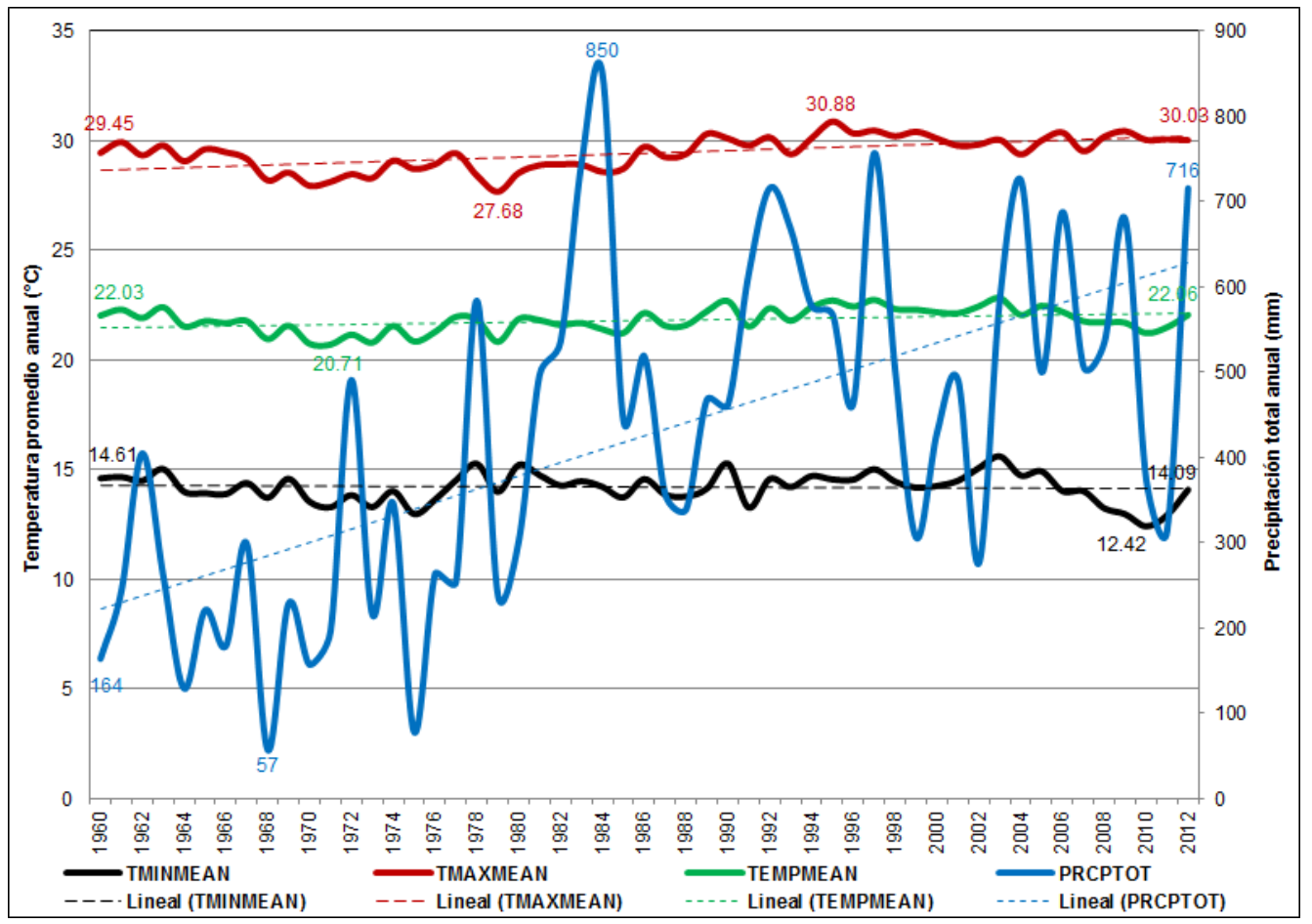

FIGURA 21 - Tendencia de la TMAXMEAN, TMINMEAN, PRCPTOT, 1960-2012. Fuente: Elaboración propia en RClimDex, con datos de CONAGUA

En cincuenta años, la tendencia del valor acumulado que tuvo la temperatura máxima media (TMAXMEAN) fue de incremento, al ser de $0.58{ }^{\circ} \mathrm{C}$, así de igual manera para el caso de la temperatura mínima media (TMINMEAN) la tendencia del valor acumulado fue a la baja, con una disminución de $0.52{ }^{\circ} \mathrm{C}$.

$\mathrm{Si}$ se analiza el promedio de ambas temperaturas (TMAXMEAN $Y$ TMINMEAN), los resultados alcanzados en este estudio indican que en las últimas cinco décadas la tendencia es de aumento de la temperatura media acumulada en $0.03^{\circ} \mathrm{C}$ en la Reserva de la Biosfera El Vizcaíno, B.C.S. 


\section{CONCLUSIONES}

La mayoría de los índices obtenidos y analizados para la Reserva de la Biosfera el Vizcaíno REBIVI presentaron tendencia de aumento. La mayoría de los índices basados en la temperatura mostraron patrones de comportamientos similares y consistentes con tendencia general de aumento en la temperatura en las últimas cinco décadas.

El $75 \%$ de los índices de cambio climático analizados mostraron una tendencia positiva, siendo estos:

- $\quad$ Longitud máxima de la racha lluviosa (CWD)

- $\quad$ Días de precipitación abundante (R20MM)

- $\quad$ Indicador de duración de racha cálida (WSDI)

- $\quad$ Rango diurno de temperatura (DTR)

- $\quad$ Días de verano (SU25)

- $\quad$ Noches tropicales (TR20)

- Temperatura máxima extrema (TXX)

- $\quad$ Temperatura máxima más baja (TXN)

- Temperatura mínima más alta (TNX)

- $\quad$ Noches frías (TN10P)

- $\quad$ Temperatura máxima promedio (TMAXMEAN)

- $\quad$ Precipitación total (PRCPTOT)

Y los índices con tendencia de disminución o negativa, con el $25 \%$ :

- $\quad$ Temperatura mínima extrema (TNN)

- Días consecutivos sin Iluvia (CDD)

- $\quad$ Noches cálidas (TN90P)

- $\quad$ Temperatura mínima promedio (TMINMEAN)

La metodología aplicada en este estudio puede ser reproducida en otras áreas con características semejantes.

Debido a que las series de datos son relativamente cortas (53 años), o que probablemente pueden estar bajo efectos de fenómenos atmosféricos de escala decadal que den sesgo en los resultados, no se pudo establecer con certeza la tendencia real del comportamiento de la temperatura y precipitación, por lo cual lo recomendable sería trabajar con series lo más extensas posibles.

Los índices climáticos presentan resultados muy importantes, que servirán de base para realizar el diagnóstico de vulnerabilidad presente y futura y elaborar el programa de adaptación al cambio climático de la Reserva de la Biósfera el Vizcaíno, un instrumento necesario para la conservación de la biodiversidad y aseguramiento de la continuidad de los servicios ecosistémicos, base para las actividades productivas de las comunidades en REBIVI. 
A pesar de que en la REBIVI la tendencia en cincuenta años ha sido de incremento en la precipitación, la condición de aridez en la zona está determinada por la influencia de la celda semipermanente de alta presión del Pacífico nororiental, provocado por masa de aire frío que afecta a la presencia de lluvias, y por otro lado la corriente oceánica fría de California.

\section{AGRADECIMIENTOS}

Los autores agradecen el Programa de las Naciones Unidas para el Desarrollo (PNUD) por ser patrocinador del proyecto Programa de Adaptación al Cambio Climático de la Reserva de la Biósfera El Vizcaíno (REBIVI).

\section{REFERENCIAS BIBLIOGRÁFICAS}

Acquaotta, F., Fratianni, S. and Garzena, D. Temperature changes in the North Western Italian Alps from 1961 to 2010. Theor Appl Climatol. 2015, 122:619634.

Alexander, L. V.; Zhang, X. and Peterson, T. C. Global observed changes in daily climate extremes of temperature and precipitation. Journal of Geophysical Research, 2006, 111:1-22.

Alvarez-Castañeda, S. T., C. A. Salinas-Zavala y F. de Lachica, 1995. Análisis biogeográfico del noroeste de México con énfasis en la variación climática y mastozoológica. Acta Zoológica Mexicana, nueva serie (66), 59-86 pp.

Arnone, J. A., Verburg, P. S. J., Johnson, D. W. et al. Prolonged suppression of ecosystem carbon dioxide uptake after an anomalously warm year. Nature, 2008, 455(7211):383-386.

Cavazos, T., Arriaga-Ramírez, S. Downscaled Climate Change Scenarios for Baja California and the North American Monsoon during the Twenty-First Century. J. Climate. 2012, 25(17):5904-5915.

CONANP. 2000. Programa de Manejo Reserva de la Biosfera El Vizcaíno, SEMARNAP, Instituto Nacional de Ecología, México, pp. 244.

Degefie, T., Fleischer, E., Klemm, O. et al. Climate extremes in South Western Siberia: past and future. Stoch Environ Res Risk Assess. 2014, 28:2161-2173.

Englehart, P. J., Douglas, A. Changing behavior in the diurnal range of surface air temperatures over Mexico. Geophys Res Lett. 2005, 32:1-4.

Garcia, E., P. Mosiño, 1968. Los climas de Baja California. En: Comité Nacional Mexicano para el Decenio Hidrológico Internacional, Instituto de Geofísica, UNAM. Memoria 1966-1967. Editor: Ing. R. del Arenal C., México, D.F. 29-56 pp., 1 mapa.

Gutiérrez-Ruacho, O. G., Brito-Castillo, L., Díaz-Castro, S., Watts, C. Trends in rainfall and extreme temperatures in northwestern Mexico. Clim Res., 2010, 42:133-142.

INECC (Instituto Nacional de Ecología y Cambio Climático).

http://www2.inecc.gob.mx/publicaciones/libros/451/cap3.html, fecha de consulta: marzo de 2017. 
IPCC (Intergovernmental Panel on Climate Change). Fifth Assessment Report (AR5). Climate Change 2014. Synthesis Report, Geneve: www.ipcc.ch

IPCC (Intergovernmental Panel on Climate Change). Summary for policymakers of Climate Change, The physical science basis. In: contribution of working group I to the fourth assessment report of the intergovernmental panel on climate change. 2007, Cambridge University Press, Cambridge, UK. 102 p.

Rodríguez-Moreno, Víctor M., de la Mora Orozco, C., et al. Bosque mesófilo de montaña en: La vegetación de México. 1978, Limusa, México, D. F.

Magaña, V., Zermeño, D., Neri, C. (2012). Climate change scenarios and potential impacts on water availability in northern Mexico climate research Clim. Res. 51:171-184.

Martone, de E., 1926. Areisme et indice d'aridité, comptes rendus de L'Academie des Sciences de Paris 182:1393-1398, in English: Regions of Interior-Basin Drainage. The Geographical Review. Vol. 17, 397-414 pp.

National Oceanic and Atmospheric Administration (NOAA), https://www.esrl.noaa.gov/psd/data/gridded/data.noaa.ersst.v4.html, fecha de consulta: marzo de 2017.

Pavía, E. G., Graef, F., Reyes, J. (2008). Annual and seasonal surface air temperature trends in México. Int. J. Climatol. 29:1324-1329.

Rzedowski, J. 1978. Bosque mesófilo de montaña en: La vegetación de México. Limusa, México, D. F.

Sheinbaum J. Currenttheorieson. El Niño-SouthernOscillation: A review. Geofísica Internacional, 2003, Volúmen 42: 291-305.

UNESCO. 2016. Lista del Patrimonio Mundial. [En línea], consultado 17/05/2016. http://whc.unesco.org/en/interactive-map/

Vázquez, A. J. L. Guía para el cálculo y uso de índices de cambio climático en México. Instituto Nacional de Ecología. 2010, México. 376 p.

Wang, X. L. Penalized maximal $F$ test for detecting undocumented mean shift without trend change. J. Atmospheric Oceanic Technol. 2008, 25:368-384.

Wang, X. L. and Feng, Y. Rhtests V3. User manual. climate research division. atmospheric science and technology directorate. 2010, Science and technology branch. Environment Canada. 27 p.

$\mathrm{Yu}, \mathrm{Z}$. and $\mathrm{Li}, \mathrm{X}$. Recent trends in daily temperature extremes over northeastern China (1960-2011). Quaternary International, 2015, 380-381:35-48.

Zhang, X. and Feng, F. RClimDex (1.0). Manual del usuario. Climate Research Branch Environment Canada. 2004,Versión en español: Santos, J. L. CIIFEN. 22 p.

Zhang, X.; Alexander, L.; Hegerl, G. C.; Jones, P.; Klein-Tank, A.; Peterson, T. C.; Trewin, B. and Zwiers, F. W. WIREs Clim Change. 2015, Doi: 10.1002/wCc. 147. 